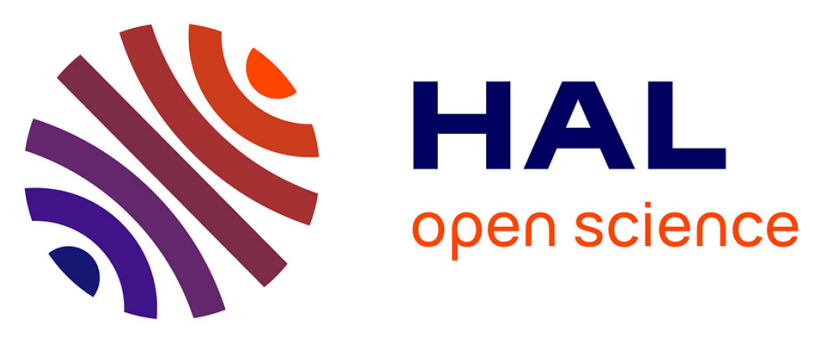

\title{
Large-Scale Modeling Approach Reveals Functional Metabolic Shifts during Hepatic Differentiation
}

Nathalie Poupin, Anne Corlu, Nicolas J. Cabaton, Hélène

Dubois-Pot-Schneider, Cécile Canlet, Elodie Person, Sandrine S. Bruel, Clément Frainay, Florence Vinson, Florence Maurier, et al.

\section{To cite this version:}

Nathalie Poupin, Anne Corlu, Nicolas J. Cabaton, Hélène Dubois-Pot-Schneider, Cécile Canlet, et al.. Large-Scale Modeling Approach Reveals Functional Metabolic Shifts during Hepatic Differentiation. Journal of Proteome Research, 2019, 18 (1), pp.204-216. 10.1021/acs.jproteome.8b00524 . hal-01937261

\section{HAL Id: hal-01937261 \\ https://hal-univ-rennes1.archives-ouvertes.fr/hal-01937261}

Submitted on 3 Dec 2018

HAL is a multi-disciplinary open access archive for the deposit and dissemination of scientific research documents, whether they are published or not. The documents may come from teaching and research institutions in France or abroad, or from public or private research centers.
L'archive ouverte pluridisciplinaire HAL, est destinée au dépôt et à la diffusion de documents scientifiques de niveau recherche, publiés ou non, émanant des établissements d'enseignement et de recherche français ou étrangers, des laboratoires publics ou privés. 


\section{A large-scale modelling approach reveals functional}

3 Nathalie Poupin*1, Anne Corlu², Nicolas J. Cabaton ${ }^{1}$, Hélène Dubois-Pot-Schneider² ${ }^{2}$, Cécile

$4 \quad$ Canlet $^{1}$, Elodie Person ${ }^{1}$, Sandrine Bruel ${ }^{1}$, Clément Frainay ${ }^{1}$, Florence Vinson ${ }^{1}$, Florence

5 Maurier ${ }^{1}$, Fabrice Morel ${ }^{2}$, Marie-Anne Robin $^{2}$, Bernard Fromenty ${ }^{2}$, Daniel Zalko ${ }^{1}$ and Fabien

Jourdan $^{1}$

8 1UMR1331 Toxalim (Research Centre in Food Toxicology), Université de Toulouse, INRA, ENVT, INP-Purpan, UPS, Toulouse, France

\section{Corresponding Author}

16 *E-mail: nathalie.poupin@inra.fr 


\section{ABSTRACT}

19 Being able to explore the metabolism of broad metabolizing cells is of critical importance in many 20 research fields. This article presents an original modelling solution combining metabolic network 21 and omics data to identify modulated metabolic pathways and changes in metabolic functions 22 occurring during differentiation of a human hepatic cell line (HepaRG). Our results confirm the 23 activation of hepato-specific functionalities and newly evidence modulation of other metabolic 24 pathways, which could not be evidenced from transcriptomic data alone. Our method takes 25 advantage of the network structure to detect changes in metabolic pathways that do not have gene 26 annotations, and exploits flux analyses techniques to identify activated metabolic functions.

27 Compared to usual cell-specific metabolic network reconstruction approaches, it limits false 28 predictions by considering several possible network configurations to represent one phenotype, 29 rather than one arbitrarily selected network. Our approach significantly enhances the 30 comprehensive and functional assessment of cell metabolism, opening further perspectives to 31 investigate metabolic shifts occurring within various biological contexts.

32 KEYWORDS: Genome scale metabolic modelling / global metabolic shifts / HepaRG cell line / 33 hepatic differentiation / transcriptomics and metabolomics 


\section{INTRODUCTION}

35 Getting a global picture of how cell metabolism is reprogrammed or shifted under different 36 conditions is a crucial step towards the understanding of how cellular metabolic functionalities can

37 be altered or modified under the induction of internal processes (e.g., cell differentiation) or 38 external factors (e.g., toxics or drugs). Technological advances in high-throughput DNA and RNA

39 sequencing methods now enable the analysis of the complete genome or transcriptome of a given

40 cell or organism, providing a first large-scale picture of its biological status under a given condition.

41 However, this information is often not sufficient to describe nor predict the metabolic phenotype

42 of the studied system, as metabolic processes involve many interconnected reactions, whose final

43 resulting activities are not always directly and strictly related to the observed gene expression

44 levels. Understanding the metabolic behavior of cells and tissues in given conditions requires

45 apprehending this complex network of reactions at the whole genome scale.

46 In this perspective, genome-scale metabolic network reconstructions (GSMNR) open promising

47 possibilities for shaping a comprehensive picture of the metabolism and its modulation within

48 various contexts, such as development, pathological processes, or under drug exposure. These

49 reconstructions aim at assembling all the biochemical transformations known to occur in a given

50 organism, based on its genome annotation and data available from the literature ${ }^{1}$. They include

51 information about the gene-protein-reactions (GPR) associations, which describe the relationships

52 between the metabolic reactions, the enzymes that catalyze them, and the genes these enzymes are

53 encoded by. Hence, GSMNR provide a relevant scaffold for the mechanistic analysis and

54 interpretation of gene expression profiles in terms of metabolic phenotype. To date, several generic

55 reconstructions have been published for the human metabolic network (Recon $1^{2}$; EHMN ${ }^{3}$; HMR2

56 4; Recon $2{ }^{5}$; Recon2.1 ${ }^{6}$; Recon $2.2^{7}$; iHsa ${ }^{8}$; Recon 3D ${ }^{9}$ ). These reconstructions represent the

57 overall theoretical metabolic capacity of a generic human cell or tissue, regardless of external 
58 conditions or cell or tissue specificities, and encompass up to 13000 reactions and 3700 genes for

59 the latest reconstruction. Because of their genericity, these reconstructions cannot directly be used

60 to predict accurately cell- or tissue- specific metabolic behaviors. They need to be tailored to more

61 specific subnetworks, by identifying the reactions from the original generic GSMNR that are

62 specifically active in the studied cell, tissue or conditions. Several methods have been proposed to

63 tackle this challenge and have already succeeded in capturing the specific metabolic features of

64 tissues ${ }^{10-12}$ or cancer cells ${ }^{13-17}$. These methods use experimental data (e.g., transcriptomic or

65 proteomic data) to predict a consistent subnetwork of reactions specifically active in the studied

66 condition that best matches with the experimental data. The algorithms used for this purpose rely

67 on constraint-based modeling (CBM) approaches, which aim at calculating the steady-state fluxes

68 of metabolites, i.e., the rates of metabolic conversion or transfer, through each network reaction ${ }^{18}$.

69 In this study, we propose a generic approach, based on the human GSMNR Recon 2, and on

70 transcriptomic and metabolomic data, to identify global changes in metabolic functions between

71 two cell populations. We based our new method on a previously published algorithm for tissue-

72 specific network reconstruction, the iMat algorithm ${ }^{10,19}$, with the originality that we depicted the

73 metabolism of the each cell population by a set of subnetworks rather than one unique subnetwork.

74 We hypothesize that this approach enables to better characterize the whole metabolic capability of

75 a cellular system independently of specific conditions and limits the risk of false predictions. We

76 applied this approach for comparing the metabolism of the human hepatic cells HepaRG in two

77 distinct differentiation stages: undifferentiated progenitor cells and fully differentiated hepatocyte-

78 like cells. The HepaRG cell line, which is derived from a human hepatocellular carcinoma, is a

79 relevant human model to study hepatic cell differentiation, as it has the particularity to exhibit

80 features of bipotent progenitor cells but is also able to differentiate into mature hepatocyte-like

81 cells. These fully differentiated hepatocyte-HepaRG cells have been demonstrated to display 
82 metabolic capacities close to primary human hepatocytes, and in particular, to express numerous 83 xenobiotic metabolizing enzymes ${ }^{20,21}$ and to present functional mitochondria ${ }^{22}$. Hepatic cell

84 differentiation is a key process in liver maintenance and regeneration and many liver diseases may 85 imply, to some extent, hepatic regenerative processes. Therefore, being able to compare the 86 metabolic capabilities of cells at different developmental stages in in vitro models is a critical step 87 when aiming to generate fully functional hepatocytes for liver transplantation or to study disease 88 or toxicological mechanisms ${ }^{23}$. Metabolic differences between pluripotent stem cells and 89 differentiated cells have been reported ${ }^{24-26}$, but studies providing a more global overview of the 90 metabolic shifts occurring during re-programming and differentiation are still lacking. Besides, 91 most studies investigating the differences between progenitors and differentiated HepaRG cells

92 have so far focused on a set of selected transcripts or proteins associated with the expression of 93 enzymes involved in xenobiotics metabolism or with liver-specific functions (such as albumin 94 production) $20,27,28$. A few global methylomic or transcriptomic analyses have enabled to further 95 explore the modulations occurring at the regulation level upon differentiation and have reported 96 the modulation of global cellular processes (such as cell cycle, cell death, apoptosis, cell 97 morphology .... $)^{29,30}$. However, information is still missing about the modulations occurring at the 98 metabolic level and involving sustainable changes in metabolic capacities and functionality of these 99 cells. The global approach we propose in this study intends to help generating a global picture of 100 the metabolism of HepaRG cells. In comparison to usual transcriptomic analyses, the method we 101 developed takes advantage of the network structure to go beyond the descriptive analysis based on 102 metabolic pathways and to gain information about the functional capacity of the cells, without 103 focusing on one a priori defined restricted set of metabolic features or genes. 
105

106

107

108

109

110

$111 \mathrm{U} / \mathrm{ml}$ penicillin and $100 \mu \mathrm{g} / \mathrm{mL}$ streptomycin, $5 \mu \mathrm{g} / \mathrm{mL}$ insulin, $2 \mathrm{mM}$ glutamine (Sigma, Saint-

112 Quentin Fallavier, France) and $50 \mu \mathrm{M}$ hydrocortisone (Serb, Paris, France). Undifferentiated

113 HepaRG cells were collected after 3 days of culture on this medium ("3-day cells"). To obtain

114 differentiated HepaRG cells, cells were cultured in the initial medium for 2 weeks, then switched

115 to a medium supplemented with $2 \%$ DMSO (Sigma) and cultured on this supplemented medium

116 for 2 more weeks. Addition of DMSO induces of HepaRG cells to two different cell types:

117 hepatocyte-like cells and biliary like-cells. HepaRG cells corresponding to fully differentiated

118 hepatocytes were called "30-day cells".

\section{Microarray analyses}

120 Total messenger RNA (mRNA) from four biological replicates was extracted from 3-day and 30121 day HepaRG cells. mRNA was checked for purity and integrity, using an Agilent Bioanalyser 122 (Agilent Technologies, Palo Alto, CA). Genome-wide expression profiling was performed using 123 the low-input QuickAmp labeling kit and human SurePrint G3 8x60K pangenomic microarrays 124 (Agilent Technologies, Santa Clara, CA, USA). Gene expression data were processed using Feature 125 Extraction and GeneSpring software (Agilent Technologies).

126 Genes were classified in 2 categories according to their expression level. Genes whose expression 127 level was below the defined threshold of 150 were considered as not detected or not expressed 128 (NE), whereas genes whose expression level was above 150 for all replicates were considered as 
129 significantly expressed (HE). In addition, genes that were found to be significantly up-regulated

130 between $\mathrm{d} 3$ and $\mathrm{d} 30$ ( $\mathrm{p}$-value $<0.01$ with an unpaired $\mathrm{t}$-test adjusted with Bonferoni correction and

131 with a fold change higher than 2) were considered as significantly expressed (HE) at d30 and,

132 similarly, down-regulated genes were considered as significantly expressed at $\mathrm{d} 3$. The threshold 133 value of 150 corresponds as the 25 percentile of the gene expression level among all genes and all 134 replicates, which is generally considered as the background noise in Agilent microarray 135 experiments.

\section{Metabolomic analyses}

137 Metabolites were extracted from 1 million of cells with an acetonitrile/water (1:9) solvent 138 mixture. Samples were centrifuged 10 minutes at 5000 g. Supernatants were evaporated using a 139 SpeedVac and resuspended in deuterated water (D20), samples were vortexed and transferred into $1405 \mathrm{~mm}$ NMR tubes.

141 1H NMR spectra of cell extracts were acquired on a Bruker Avance spectrometer (Bruker, 142 Karlsruhe, Germany) operating at $600.13 \mathrm{MHz}$, and equipped with an inverse detection $5 \mathrm{~mm}$ TXI 143 1H-13C-15N cryoprobe connected to a cryoplatform. Spectra were acquired using a Carr-Purcell144 Meiboom-Gill (CPMG) spin echo pulse sequence with a 2 seconds relaxation delay to attenuate 145 macromolecules signals. A water suppression signal was achieved by presaturation during the 146 relaxation delay. The spectral width was set to $20 \mathrm{ppm}$ for each spectrum and 512 scans were 147 collected with $32 \mathrm{~K}$ points. Free induction decays were multiplied by an exponential window 148 function before Fourier Transformation. The spectra were manually phased and the baseline was 149 corrected using TopSpin 3.2 software (Bruker, Karlsruhe, Germany).

150 Metabolites were identified using the literature, home-made and freeware databases such as the 151 Human Metabolome Database ( ${ }^{31}$; www.hmdb.ca/). 
152 Implementation and adaptation of the iMat algorithm to generate stage-specific metabolic

153 models of HepaRG cells

154 We used the generic human metabolic network reconstruction Recon 2 (5; version 2.04, 155 downloaded from http://vmh.uni.lu/\#downloadview) as a framework for the prediction of active 156 metabolic reactions in the HepaRG cells. This genome-scale metabolic network reconstruction 157 encompasses 7440 reactions, 2140 genes and 2626 unique metabolites and is supposed to represent 158 the comprehensive metabolic capacity of any human cell or tissue. The network reconstruction is 159 converted into a mathematical model, where the list of metabolic reactions is described as a 160 stoichiometric matrix (S). This matrix defines which metabolites (enumerated as rows) participate 161 in each of the network reactions (enumerated as columns), with numerical entries in the matrix 162 representing the stoichiometric coefficients of the reactions.

163 First, gene expression data were mapped to the network reactions by using the Gene-Protein164 Reaction (GPR) association rules defined in the network reconstruction. We used these GPR 165 associations to determine a set of a priori highly expressed reactions (HEr) or not expressed 166 reactions (NEr) according to the expression level of their associated gene(s). In the case of "AND" 167 GPR associations, we classified the reaction as HEr (or NEr respectively) if all genes were HE (or 168 NE respectively), whereas in the case of "OR" associations, the reaction was considered as HEr if 169 any of the associated gene was HE. In the initial Recon 2 reconstruction, 4821 reactions had defined 170 GPR associations.

171 We then implemented the iMat algorithm ${ }^{10}$, that we adapted, in order to predict which reactions 172 from the generic metabolic network Recon 2 were specifically active in the HepaRG cells (Figure 173 S1). As initially proposed by Shlomi et al., the optimization problem was formulated as a Mixed 174 Integer Linear Programming (MILP) problem, to find a steady-state flux distribution that 175 maximizes the number of reactions whose flux is consistent with the measured expression level of 
176 their associated gene(s) and that complies with stoichiometric and thermodynamic constraints. In 177 our modified version of this algorithm, we added some constraints so that the predicted flux 178 distribution is also in agreement with the metabolomics data, meaning that it allows all detected 179 metabolites to be produced. More precisely, for each identified metabolite, at least one reaction 180 that is able to produce it in the model should have a non-zero flux.

$181 \quad \max \sum_{i \in H E_{r}}\left(y_{i}^{+}+y_{i}^{-}\right)+\sum_{i \in N E_{r}} y_{i}$

$182 v, \mathrm{y}^{+}, \mathrm{y}^{-}, \mathrm{y}$

183 subject to

$184 \quad$ S. $v=0$

185

186

187

188

189

$$
v_{\min } \leq v \leq v_{\max }
$$

$$
v_{i}+y_{i}^{+}\left(v_{\min , i}-\varepsilon\right) \geq v_{\min , i}, \mathrm{i} \in \mathrm{HEr}
$$

$$
v_{i}+y_{i}^{-}\left(v_{\max , i}+\varepsilon\right) \leq v_{\max , i}, i \in \operatorname{HEr}
$$

$$
v_{i}+y_{i} \cdot v_{\min , i} \geq v_{\min , i}, \mathrm{i} \in \mathrm{NEr}
$$

$$
v_{i}+y_{i} \cdot v_{\max , i} \leq v_{\max , i}, \mathrm{i} \in \mathrm{NEr}
$$

$v$ is the flux vector and $\mathrm{S}$ is the stoichiometric matrix.

195 Equation (1) ensures that flux values comply with the mass balance constraints. Thermodynamic 196 and capacity constraints are imposed by equation (2): restricted direction and values for reaction 197 flux, according to these constraints, are defined in $v_{\min }$ and $v_{\max }$ vectors (minimal and maximal flux 
198 values respectively), which are set as lower and upper bound for reaction flux values. Equations

199 (3) to (6) set constraints corresponding to gene expression data. $\mathrm{y}_{\mathrm{i}}^{+}, \mathrm{y}_{\mathrm{i}}^{-}$and $\mathrm{y}_{\mathrm{i}}$ are boolean variables 200 representing the adequacy between the predicted flux $v_{\mathrm{i}}$ through reaction $\mathrm{i}$ and its expression level.

$201 \mathrm{For} \mathrm{HEr}_{\mathrm{i}}^{+}\left(\mathrm{y}_{\mathrm{i}}^{-}\right)$represents whether the reaction is active in the forward (or backward, respectively) 202 direction: equations (3) \& (4) enforce that, if $\mathrm{y}_{\mathrm{i}}^{+}=1$ or $\mathrm{y}_{\mathrm{i}}^{-}=1$ (i.e., the reaction is active), the value 203 of the flux through reaction $\mathrm{i}$ is larger than a threshold $\varepsilon$, whereas if $\mathrm{y}_{\mathrm{i}}^{+}=0$ or $\mathrm{y}_{\mathrm{i}}^{-}=0$ (i.e., the reaction 204 is inactive), the flux through reaction i must be 0 . For NEr, $y_{i}$ represents whether the reaction is 205 inactive: equations (5) \& (6) enforce that, if $\mathrm{y}_{\mathrm{i}}=1$ (i.e., the reaction is inactive), the flux through 206 reaction $i$ is 0 . A threshold of 0.1 was used to predict the activity of reactions. Different threshold 207 values were tested and provided qualitatively similar results. Equations (7) to (9) set constraints 208 corresponding to metabolomic data. For each identified metabolite j ("ExpMets"), "RProd ${ }_{j} "$ 209 encompass all reactions $i$ that can produce this metabolite according to the stoichiometric matrix 210 S. $\mathrm{x}_{\mathrm{i}}^{+}$(or $\mathrm{x}_{\mathrm{i}}^{-}$, respectively) are Boolean variable representing whether the reaction $\mathrm{i}$ is active or not 211 in the forward (or backward, respectively) direction. When $\mathrm{x}_{\mathrm{i}}^{+}=1$, the flux through reaction i must 212 be larger (or smaller, respectively) than $\varepsilon$ (equation (7)), forcing the reaction to be forward active.

213 For reversible reactions, when $\mathrm{x}_{\mathrm{i}}^{-}=1$, the flux through reaction $\mathrm{i}$ must be negative and lower than $214 \varepsilon$ (equation (8)), forcing the reaction to be backward active. If $\mathrm{x}_{\mathrm{i}}^{+}=0$ and $\mathrm{x}_{\mathrm{i}}^{-}=0$, the flux through 215 reaction $\mathrm{i}$ is forced to be 0 , forcing the reaction to be inactive. The constraints set in Eq. 9 ensure 216 that at least one reaction is able to produce the metabolite $\mathrm{j}$ is active.

217 The optimization problem consists in finding the flux distribution $v$, which maximizes the 218 number of $\mathrm{HEr}$ which are active $\left(v_{\mathrm{HEr}} \geq \varepsilon\right)$ and the number of NEr which are inactive $\left(v_{\mathrm{NEr}}=0\right)$.

219 This sum represents the "adequacy score" of the flux distribution. The percentage of adequacy is 220 calculated as the adequacy score divided by the theoretical maximal adequacy score that could be 
221 obtained (i.e., the sum of all reactions associated with HE genes and the sum of all reactions 222 associated to NE genes).

223 The algorithm was implemented in Matlab 2014a (The Mathworks, Natick, MA, USA) and the 224 resolution of the MILP problem was performed using the ilog CPLEX solver version 12.6.0 (ILOG, 225 Sunnyvale, CA, USA).

226 Identification of alternative adequate stage-specific subnetworks equally fitting the 227 experimental data

228 The flux distribution obtained by solving the MILP problem is optimal in term of adequacy with 229 transcriptomic and metabolomic data, but is not unique. Alternative solutions exist that have the 230 same adequacy with experimental data. To explore the space of alternative solutions, as proposed 231 by Shlomi et al. and in a further study ${ }^{32}$, we searched whether, for each reaction that was predicted 232 to be active (or inactive, respectively) in the first optimal solution, a solution with a similar 233 adequacy score could be found with this reaction being inactive (or active, respectively) (Figure 234 S1C). From the initial computed flux distribution, each reaction was successively forced to be 235 either active (i.e., to carry a non-zero flux, with $v \geq \varepsilon)$ or inactive $(v=0)$ and a new MILP 236 optimization was performed. Reversible reactions were successively set to be active in each 237 direction $(v \geq \varepsilon$, then $v \leq-\varepsilon$ ). Only flux distributions having a maximal adequacy score were kept, 238 forming the final set of optimal solutions. Each of these solutions represents a subnetwork of 239 predicted active reactions, with optimal adequacy with experimental data. The same computations 240 were performed independently using the data obtained at $\mathrm{d} 3$ and $\mathrm{d} 30$, providing one set of equally 241 adequate subnetworks with regard to gene expression data for each $\mathrm{d} 3$ and $\mathrm{d} 30$ stage.

242 For each stage, reactions that were found to be active in all possible subnetworks were considered 243 as "required" (R) reactions, meaning that no maximal adequacy score could be achieved when these 244 reactions were forced to be inactive. Conversely, reactions that display a zero-flux in all the optimal 
245 solutions were considered as "Inactive" (I) reactions, since no maximal adequacy score could be

246 achieved when these reactions were forced to be active. All other reactions that are either active or

247 inactive in the optimal solutions were considered as "Potentially active" (PA) reactions. As a result,

248 sets of R, PA and I reactions were defined for each stage (Figure S1D).

249 Assessment of the liver-specificity of genes in generated subnetworks

250 To evaluate the liver-specificity of our generated models, we assessed, for each subnetwork, the 251 number of predicted "expressed" genes that can be considered as liver-specific. We used 252 information about the tissue location of genes from the UNIPROT ${ }^{33}$ and the Human Protein Atlas $253\left(\mathrm{HPA}^{34}\right)$ databases.

254 First, for each generated subnetwork, genes predicted as "expressed" were inferred from the 255 predicted active reactions using the GPR rules. UNIPROT identifiers for Recon 2 genes were 256 retrieved from their EntrezGene identifiers (as available in the initial reconstruction) using the

257 DAVID database ${ }^{35}$ and predicted "expressed" genes were compared to genes with evidence for 258 presence in the liver according to the UNIPROT database. For each subnetwork, we calculated the 259 "recall" of liver-specific genes, as the proportion of Recon 2 genes with evidence of presence in 260 liver that were predicted to be "expressed" in the subnetwork, and the "precision", as the proportion 261 of predicted "active" genes in the subnetwork that were liver located.

262 In a second analysis, we mapped the confidence level for protein expression in liver hepatocytes 263 assigned in the HPA database (High, Medium, Low or Not detected) to the generated subnetworks 264 and evaluated, in our HepaRG 3-day and 30-day subnetworks, the proportion of predicted active 265 reactions associated with high, medium or low confidence level for protein expression in liver 266 according to the HPA database. The recall of reactions associated with high confidence for liver 267 expression was calculated as the proportion of reactions with high confidence level that were 
268 predicted to be active in each subnetwork. The precision was calculated as the proportion of

269 predicted active reactions that had a high confidence level.

\section{Simulation of achievable metabolic functions in generated subnetworks}

271 We performed flux balance analysis to test whether our generated subnetworks were able to 272 achieve some defined metabolic functions. A list of 155 metabolic functions (SI Table S1), 273 including 111 generic functions (i.e., functions assumed to be fulfilled by any tissue or cell 274 independently of its type) and 44 hepato-specific functions, was gathered from previous 275 publications ${ }^{4,36,37}$. For each of these tasks, we defined a corresponding objective function and 276 maximized it to check whether it could carry a non-zero flux. The sets of metabolites that could be 277 taken up and released was restrained to mimic a minimal standard medium and adjusted depending 278 on the specific metabolic objective tested. For instance, we tested the ability of our models to 279 perform gluconeogenesis (the formation of glucose from various gluconeogenic substrates) by

280 restraining the set of metabolites that could be taken up to only non-carbon sources $\left(\mathrm{O}_{2}\right)$ except for 281 lactate and glucogenic amino acids (alanine, glutamine ...) and maximize the excretion of glucose.

282 This functional testing was performed for each of the generated subnetworks at both $\mathrm{d} 3$ and $\mathrm{d} 30$.

283 We verified that all the 155 defined metabolic functions could be achieved by the initial Recon 2 284 model. The complete list of tested metabolic functions and corresponding applied constraints for 285 uptake and secretion is provided in supplemental Table 1.

286 Analysis of the variability among generated subnetworks

287 PCA was performed on all generated subnetworks, independently of the stage. Reactions were 288 used as variables and each subnetwork was represented by a vector of binary values corresponding 289 to the predicted activity state of each reaction ( 0 for predicted inactive reactions and 1 for predicted 290 active reactions).

291 Pathway enrichment analyses for identification of activated and inactivated pathways 
292 Reactions were considered as inactivated during the differentiation if they were predicted to be

293 active in at least one subnetwork (R or PA) at d3 and inactive at d30, and conversely activated if

294 they were predicted to be inactive at $\mathrm{d} 3$ and R or PA at d30. Pathway enrichment analyses were

295 performed over activated and inactivated reactions to assess whether the given reactions were

296 significantly over-represented in a metabolic pathway. Pathway enrichment statistics were

297 performed using one-tailed exact Fisher test, with a Bonferroni correction for multiple tests ${ }^{38}$,

298 using the metabolic pathways defined in Recon 2.04. Blocked reactions, identified using flux

299 variability analysis, were excluded from the background set of Recon 2 reactions. Pathway

300 enrichment analyses were also performed for the sets of highly or not expressed genes.

301 Assessing the benefits of the developed approach: comparison with predictions made from

302 transcriptomic data only

303 To assess the interest of using the network topology and stoichiometry to predict the metabolic

304 modulations occurring during the differentiation process, we performed pathway enrichment

305 analyses on genes identified as up or downregulated between the two stages. Genes were

306 considered as upregulated if they were classified as NE at d3 and HE at d30 and inversely,

307 downregulated, if they were classified as $\mathrm{HE}$ at $\mathrm{d} 3$ and $\mathrm{NE}$ at $\mathrm{d} 30$.

308 Assessing the benefits of the developed approach: comparison with predictions made from 309 single solutions

310 We compared our predictions with predictions that would be obtained when considering only

311 one individual optimal subnetwork instead of a set of optimally adequate subnetworks for each

312 stage. For each stage, we selected two distinct individual solutions "iMat-A" and "iMat-B" among

313 all the equally adequate generated subnetworks. "iMat-A" is the first initial optimal solution,

314 corresponding to the one returned by the iMat algorithm such as implemented in the CobraToolbox.

315 "iMat-B" is one other randomly selected solution among all optimal solutions. We also compared 
316 our results with results obtained by using the FASTCORE algorithm ${ }^{39}$, which provides with one

317 particular solution minimizing the number of active reactions ("FASTCORE solution"). For each

318 stage, we used the set of reactions associated with highly expressed genes as input for the "core

319 reactions set" in the FASTCORE algorithm, which then returns a minimal flux consistent 320 subnetwork containing all the reactions from this core set and a minimal set of additional reactions.

321 To compare individual solutions to the whole set of subnetworks, we considered the union of

322 subnetworks. For liver-specific genes, we considered all the genes that were predicted to be 323 expressed in any of the cell-specific subnetworks. For reactions predicted to be activated in the

324 union of equally adequate subnetworks, we considered all the reactions that were predicted to be 325 active in any of the 30-day cell-specific subnetworks but inactive in all 3-day cell-specific 326 subnetworks. Inversely for reactions predicted to be inactivated in the union of subnetworks, we 327 considered all the reactions that were predicted to be inactive in any of the 30-day subnetworks but 328 active in all 3-day subnetworks.

$329 \quad$ Data availability

330 Gene expression data have been deposited in the Gene Expression Omnibus (GEO) database 331 under the accession number GSE112123. NMR metabolomic data are provided as Supporting 332 Information (Table S2). 


\section{RESULTS}

335 In this study, we aimed at comparing the differences in metabolic functions expressed in 3-day 336 (non-differentiated) progenitor HepaRG cells ("3-day cells") vs. 30-day fully differentiated 337 hepatocyte-like HepaRG cells ("30-day cells"), using a large-scale modelling approach. To that 338 purpose, we defined consistent metabolic models that distinctively represent the functional 339 metabolism of 3-day cells and 30-day cells. We used the generic genome scale metabolic 340 reconstruction Recon $2^{5}$ as a scaffold to integrate transcriptomic and metabolomic data and 341 predicted which of the Recon 2 reactions would be specifically active or inactive at each 342 differentiation stage in HepaRG cells. Prediction of the reaction activities was computed using the 343 iMat algorithm proposed by Shlomi et al. ${ }^{10}$ that we adapted to fit the specific objectives of our 344 study. Figure S2 illustrates the pipeline followed in this study.

345 Experimental transcriptomic and metabolomic data provide only partial coverage of the 346 metabolic network

347 For each differentiation stage, we identified expressed (HE) or not expressed (NE) genes from 348 the experimental data set and transferred this information to reactions using the GPR associations. 349 As expected, only a small proportion (9\%) of the genes classified as HE or NE from the 350 experimental data set could be mapped into Recon 2, these mapped genes representing $32 \%$ of the 351 metabolic genes currently annotated in Recon 2 (Figure S3A\&B). According to GPR associations, $35229-31 \%$ of Recon 2 reactions could be linked to gene expression data (Figure S3C). More precisely, $35316 \%$ and $21 \%$ of Recon 2 reactions were associated with HE genes at day $3(\mathrm{~d} 3)$ and day $30(\mathrm{~d} 30)$, 354 respectively, whereas 13\% (d3) and 10\%(d30) were associated with NE genes. Of note, the relation 355 between reactions and genes is not bijective: one reaction can be linked to several distinct genes, 356 for instance when several isoenzymes are involved, and conversely one gene product can control 357 more than one reaction. For instance, for the carnitine palmitoyltransferase 1A gene (CPT1A, 
358 hsa:1374), the encoded protein can catalyze the transfer of the acyl group of different long-chain

359 fatty acids (FAs) onto carnitine. In parallel, NMR analyses allowed the detection and identification 360 of 36 and 34 metabolites in 3-day and 30-day HepaRG cells, respectively, out of which 34 (d3) and $36132(\mathrm{~d} 30)$ metabolites could be mapped into Recon 2 (Table S2).

362

Reconstruction of stage-specific cell models from experimental data: multiple possible

365 The cell-specific metabolic network reconstruction algorithm uses gene expression levels as

366 clues to predict the activity state of the associated reactions. It relies on CBM methods to find

367 metabolic flux distributions, which (1) maximize the number of reactions whose predicted flux is

368 consistent with associated gene expression level and (2) comply with the stoichiometric constraints

369 imposed by the network topology. We adapted the iMat algorithm so that the predicted flux

370 distributions would also take into account metabolomics data (see Material and Methods).

371 We identified several flux distributions having a similar adequacy with the experimental gene 372 expression data, as assessed by the "adequacy score" (i.e., the proportion of reactions whose 373 predicted activity state is in adequacy with the transcriptomic data). One predicted flux distribution 374 corresponds to a set of active reactions (i.e., reactions carrying a non-zero flux) representing a 375 consistent and fully connected subnetwork. We identified 3534 distinct subnetworks having a 376 maximal adequacy score of $75 \%$ for the 3 -day cells, and 3313 distinct subnetworks with an 377 adequacy score of $72 \%$ for the 30 -day cells (Table S3). These two sets of equally optimal 378 subnetworks will be subsequently referred to as "cell-specific models". As imposed by the 379 modeling constraints, all distributions enable the production of the set of NMR identified 380 metabolites. We tested that no better adequacy with transcriptomic data could be obtained when 381 releasing the additional constraints imposed from metabolomic data (see Material \& Methods for 
382 details), which means that there was no specific disagreement between transcriptomic and

383 metabolomic data. The number of predicted active reactions was significantly higher in 30-day

384 cell-specific models than in 3-day cell-specific models, ranging from 2842 to 3036 at d30 (median:

3852931 ) and from 2445 to 2673 (median: 2567) at d3 (Figure S4). The predictions of reactions activity

386 for all subnetworks are provided in Table S4.

387 Rather than selecting only one arbitrary subnetwork, we chose to take into account all the

388 alternative solutions we found to be equally adequate with the experimental data for each

389 differentiation stage. Reactions that were consistently predicted to be active in all subnetworks for

390 one differentiation stage were considered as "required reactions" (R) whereas reactions whose

391 predicted activity varied across subnetworks were considered as "potentially active" (PA) (Figure

392 S2B). $56 \%$ and $60 \%$ of Recon 2 reactions were globally predicted to be active, either PA or R (i.e.,

393 active in at least one subnetwork), at $\mathrm{d} 3$ and at $\mathrm{d} 30$ respectively. At $\mathrm{d} 30,37 \%$ of these active

394 reactions were predicted to be required, whereas a slightly lower proportion of R reactions was

395 found at d3 (27\%) (Figure 1A). We identified two main causes contributing to the high number of

396 alternative possible subnetworks. The first one is the lack of gene expression information for some

397 reactions. Globally, we observed that reactions with a variable predicted activity state (PA

398 reactions) are associated with gene expression data in a higher proportion (2\%) than reactions with

399 consistent predicted activity among stage-specific models ( $\mathrm{R}$ reactions; 58\%). The second one is

400 the existence of several alternative reactions performing the same metabolic transformation. For

401 instance, some biochemical conversions happen to be described by two alternative paths that use a

402 different number of reaction steps or the same reactions are sometimes duplicated with more than

403 one tissue-specific annotation. As a striking example, in the N-glycan synthesis pathway of the

404 Recon 2 network, two alternative paths of 22 reactions enable the synthesis of the glycan precursor

405 before it is bound to a protein in the endoplasmic reticulum (ER) and further processed in the ER 
406 and Golgi apparatus. The two paths only differ by the fact that the reactions are annotated as liver 407 reactions ("_L") in one and as uterine reactions ("_U") in the other. These 44 reactions have been 408 identified through Principal Component Analysis (PCA) as the reactions that contribute the most 409 to the discrimination between subnetworks within each set of stage-specific subnetworks (Figure 410 S5) demonstrating that path redundancy can contribute to the variability among subnetworks.
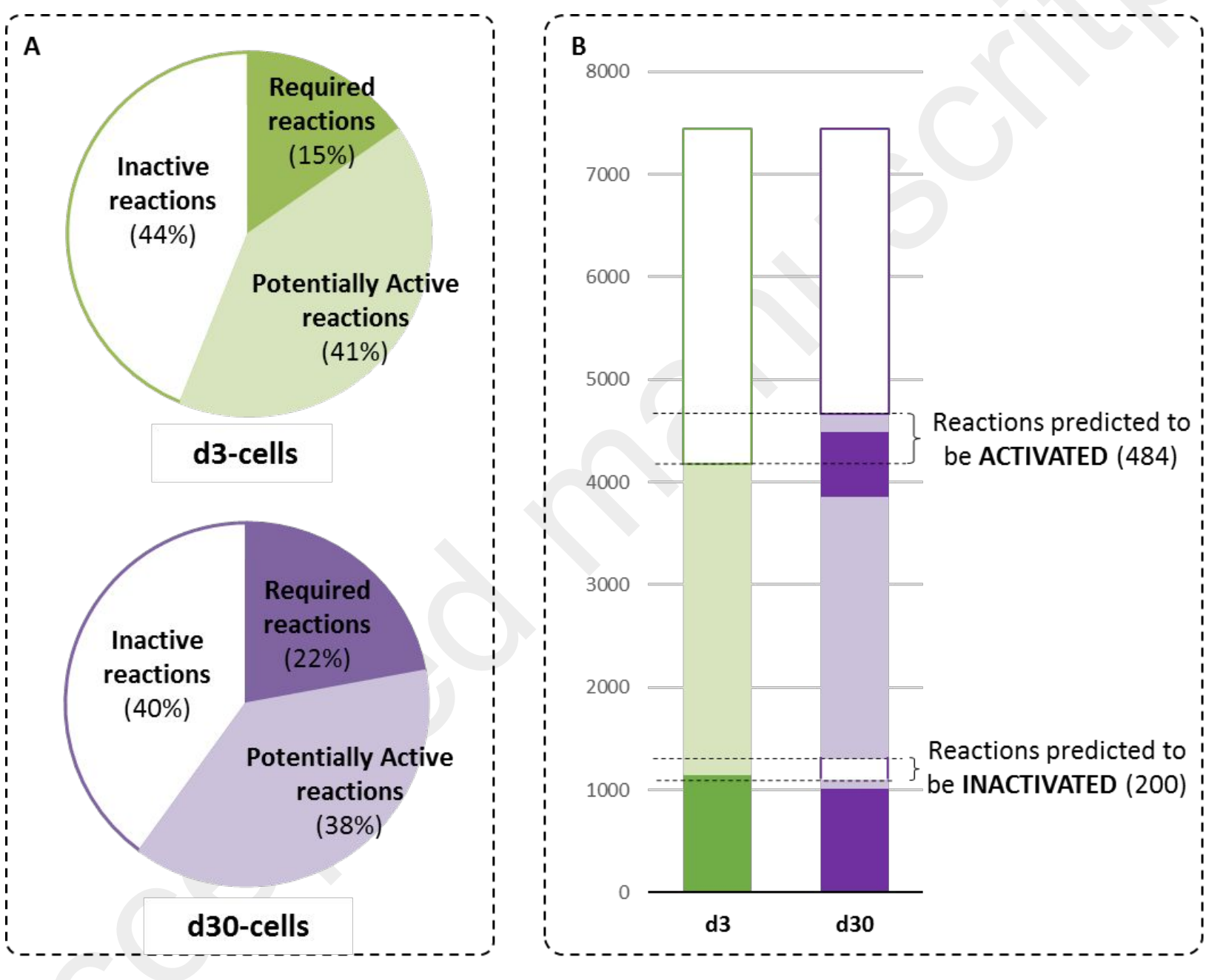

412 Figure 1. Predicted activity of Recon 2 reactions in stage-specific subnetworks.

414 Comparison of stage-specific subnetworks: evidences for global metabolic changes during

\section{5 the differentiation process}


416 To investigate which significant metabolic changes occur between 3-day and 30-day HepaRG 417 cells, we compared the cell-specific models generated for each stage. We first performed an 418 unsupervised discriminant analysis (PCA) of all the cell-specific models together. The score plot 419 of the PCA analysis showed a clear separation between the two stages, with more than $40 \%$ of the 420 variability explained by the differentiation stage represented along the first axis (Figure 2). This 421 demonstrates not only that there are metabolic differences between these two stages, but also that 422 these differences exceed the differences between the subnetworks within each stage, which is 423 represented by the second axis and accounts for only $2.5 \%$ of the total variability.

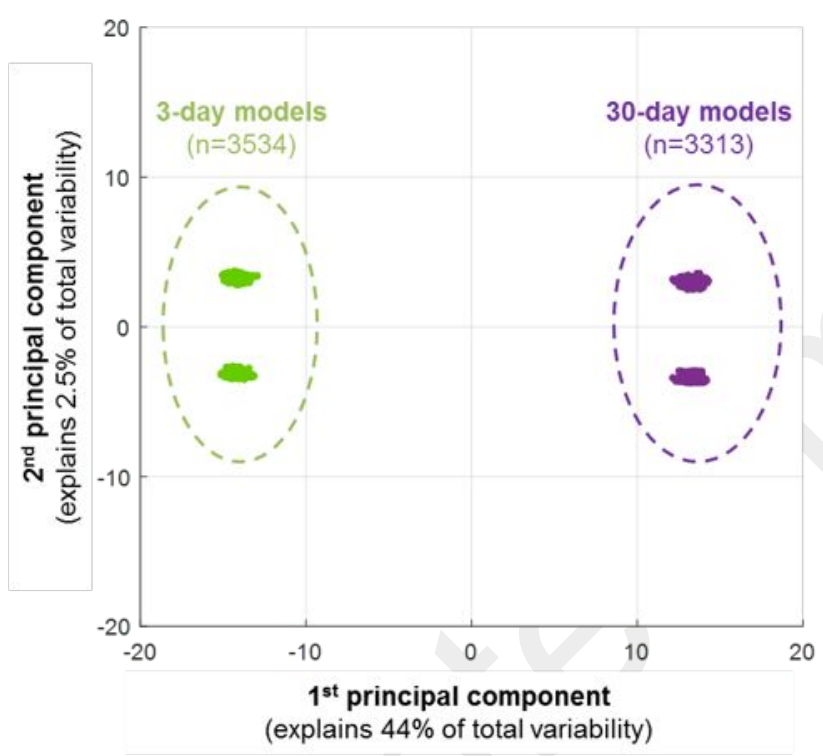
(explains $44 \%$ of total variability)

425 Figure 2. Principal component analysis of 3-day and 30-day subnetworks

426 We identified reactions that are activated during the differentiation process, by considering the 427 reactions predicted to be inactive in all $\mathrm{d} 3$ cell-specific networks but active in at least one of the 428 30-day cell-specific model (i.e., PA or R at d30). Conversely, reactions predicted to be active at $\mathrm{d} 3$ 429 but inactive at $\mathrm{d} 30$ were identified as inactivated during differentiation (Figure 1B). According to 430 our predictions, 484 reactions were activated and 200 reactions inactivated during HepaRG cell 431 differentiation. These reactions were further analyzed through pathway enrichment. Activated 
432 reactions were significantly over-represented in known hepato-specific pathways (bile acid 433 synthesis and cytochrome metabolism) but also in the following pathways: biotin metabolism, FA 434 oxidation and tryptophan metabolism. The biotin metabolism pathway acts as a cycle, where the 435 biotin binds to carboxylase enzymes (biotinylation), enabling their activation. Biotin is then 436 released by hydrolysis, to be recycled and re-used for coenzyme activity. Carboxylase enzymes 437 activated by biotinylation are involved in many important cellular functions, related in particular 438 to the production and breakdown of proteins, fats, and carbohydrates and to urea metabolism ${ }^{40}$, 439 which are indeed essential in fully functional hepatic cells. The essential amino acid tryptophan is 440 mainly metabolized in the liver through the kynurenine pathway, which accounts for about $95 \%$ of 441 its degradation in normal physiological conditions ${ }^{41}$. The prediction of the activation of this 442 pathway in mature hepatocytes is consistent with the observation of the increased activity of 443 enzymes involved in this pathway during the development in rat liver ${ }^{42}$. Conversely, the activity 444 of the extracellular transport pathway and of the FA synthesis pathway was predicted to be lower 445 in 30-day HepaRG cells (Table 1). Our results therefore suggest a shift in the metabolism of FAs, 446 with a decrease in the FA synthesis and a concomitant increase in FA oxidation in differentiated 447 cells.

448 Table 1. Predictions of metabolic pathways significantly modulated between $\mathrm{d} 3$ and $\mathrm{d} 30$

\begin{tabular}{|c|c|c|c|c|c|}
\hline \multicolumn{3}{|c|}{ ACTIVATED pathways } & \multicolumn{3}{|c|}{ INACTIVATED pathways } \\
\hline Pathways & $\begin{array}{l}\% \text { pathway } \\
\text { reactions }\end{array}$ & $\begin{array}{l}\text { corrected } \\
\text { p-value }\end{array}$ & Pathways & $\begin{array}{l}\text { \% pathway } \\
\text { reactions }\end{array}$ & $\begin{array}{l}\text { corrected } \\
\text { p-value }\end{array}$ \\
\hline Biotin metabolism & 100 & $1.31 \mathrm{e}^{-11}$ & Transport, extracellular & 9.6 & $5.60 \mathrm{e}^{-31}$ \\
\hline Fatty acid oxidation & 15.9 & $6.80 \mathrm{e}^{-07}$ & Fatty Acid Synthesis & 45.2 & $1.30 \mathrm{e}^{-15}$ \\
\hline Bile acid synthesis & 27.9 & $5.13 \mathrm{e}^{-05}$ & & & \\
\hline
\end{tabular}




$\begin{array}{lcc}\begin{array}{l}\text { Tryptophan } \\ \text { metabolism }\end{array} & 40.0 & 2.44 \mathrm{e}^{-04} \\ \begin{array}{l}\text { Cytochrome } \\ \text { metabolism }\end{array} & 57.1 & 3.76 \mathrm{e}^{-04} \\ \begin{array}{l}\text { Blood group synthesis } \\ \text { Lysine metabolism }\end{array} & 30.4 & 1.59 \mathrm{e}^{-03} \\ \begin{array}{l}\text { Limonene \& pinene } \\ \text { degradation }\end{array} & 100 & 1.44 \mathrm{e}^{-03} \\ & & 3.45 \mathrm{e}^{-02}\end{array}$

449

$450 \quad$ Visualization-based mining strengthens metabolic activity comparisons

451 Although pathway enrichment analysis offers a first global view of the pathways modulated

452 during the differentiation process, some of these pathways, such as the FA synthesis and oxidation

453 pathways, include a very large number of reactions (126 and 868 reactions, respectively). A deeper

454 examination of the reactions modulated within these pathways was carried out using the

455 MetExplore web server for visualization ${ }^{38,43}$. We observed that FA oxidation reactions predicted

456 to be activated between $\mathrm{d} 3$ and $\mathrm{d} 30$ were specifically located in the peroxisome, whereas most

457 mitochondrial FA oxidation reactions were predicted to be active at both differentiation stages

458 (Figure 3). Notably, peroxisomal FA oxidation allows the specific degradation of very long-chain

459 FAs $\left(>\mathrm{C}_{20}\right)$ whereas the shortened FAs are further oxidized in the mitochondria ${ }^{44}$. Our results

460 therefore imply that increased peroxisomal FA oxidation induced by drugs or FA overload ${ }^{45}$ cannot $^{4}$

461 occur in progenitor cells. Although limited peroxisomal FA oxidation can reduce energy

462 production, it primarily induces the accumulation of toxic very long-chain FA metabolites ${ }^{46}$.

463 Regarding the FA synthesis pathway, the visualization highlighted that reactions predicted to be

464 inactivated between $\mathrm{d} 3$ and $\mathrm{d} 30$ were more specifically involved in the elongation of the carbon

465 chain, suggesting that the synthesis of long-chain FAs cannot be achieved anymore in fully 
466 differentiated hepatocytes. However, transformation of FAs or acyl-CoA, through desaturation or

467 hydrolysis reactions respectively, can be performed at both stages of development (Figure S6). 468

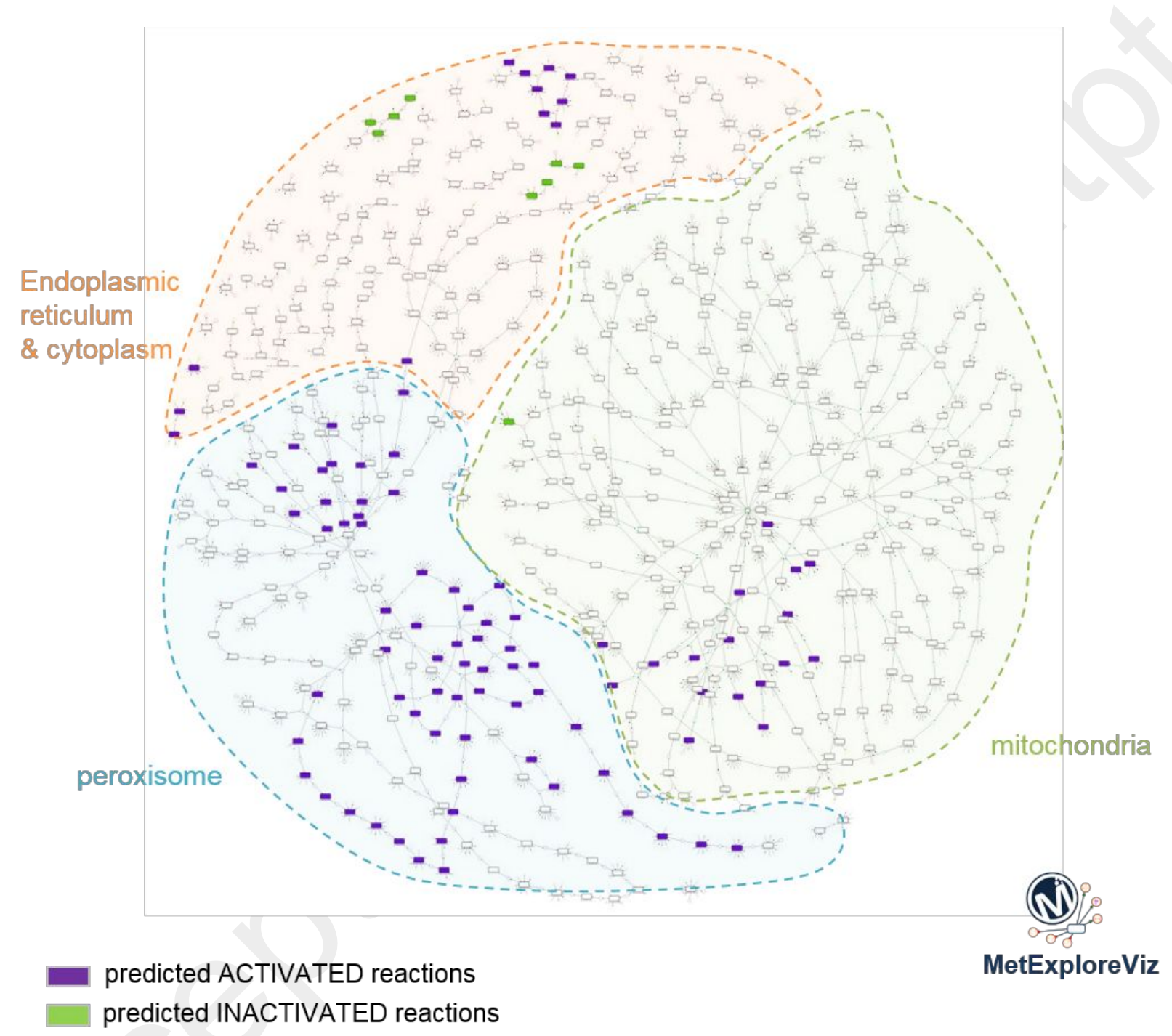

470 Figure 3. Visualization of predicted modulated reactions in the fatty acid oxidation pathway

472 Analysis of flux-consistent cell-specific models reveals functional metabolic changes during 473 the differentiation process 
474 To assess whether the generated 3-day and 30-day cell-specific models actually accounted for

475 distinct liver-specific metabolic functionalities, we challenged the capacity of each cell-specific 476 subnetwork to perform 154 defined metabolic functions ${ }^{4,36,37}$ (Table S1). Out of this list, 88

477 functions are generic functions, which can theoretically be achieved by any cell type, and 66 are 478 hepato-specific metabolic functions known to specifically take place in liver, such as ammonia 479 detoxification (through ureagenesis), ketogenesis, biliary acid formation, or gluconeogenesis. On 480 average, a higher number of tested metabolic functions, and especially of liver-specific functions, 481 could be achieved by 30-day cell-specific models compared to 3-day models: $50 \%$ vs. $31 \%$ for 482 hepatic functions and $42 \%$ vs. $33 \%$ for generic functions (Figure 4). Similarly, when comparing 483 the set of all metabolic functions that can be fulfilled by the whole set of models at each stage (i.e., 484 "UNION" of models), we observed that a larger range of hepatic functions can be fulfilled at 30 485 days than 3 days. More specifically, our models predict that 30-day cells are able to degrade a 486 wider range of amino acids and to produce the ketone body $\beta$-hydroxybutyrate compared to 3 -day 487 cells (Table 2). Interestingly, this end product of mitochondrial FA oxidation presents different 488 cellular signaling functions in addition to its role in energy production ${ }^{47}$. Regarding the 489 gluconeogenesis capacities, which were assessed by testing the ability of the models to account for 490 the production of glucose in the absence of carbohydrate sources (e.g., from non-carbohydrates 491 metabolites including lactate, pyruvate, glycerol and glucogenic amino acids), results indicate that 492 gluconeogenesis can theoretically be carried out by HepaRG cells at both stages, although by a 493 higher proportion of cell-specific models at $\mathrm{d} 30$ than $\mathrm{d} 3$. In agreement with the acquisition of liver494 specificity over the differentiation process, our models predict that only 30 -day cells can produce 495 urea from the degradation of AA (Table 2 \& Figure 5) and can synthesize bile acids such as 496 glycocholate, glycochenodeoxycholate and taurocholate. 


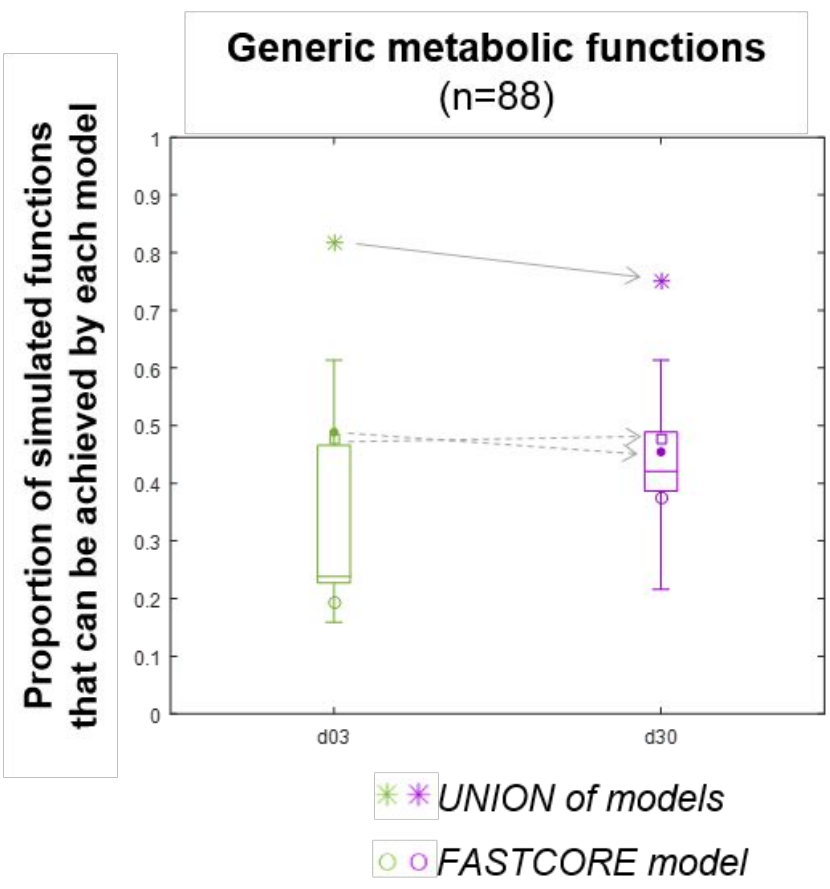

497

498 Figure 4. Simulations of metabolic functions in $\mathrm{d} 3$ and $\mathrm{d} 30$ models.

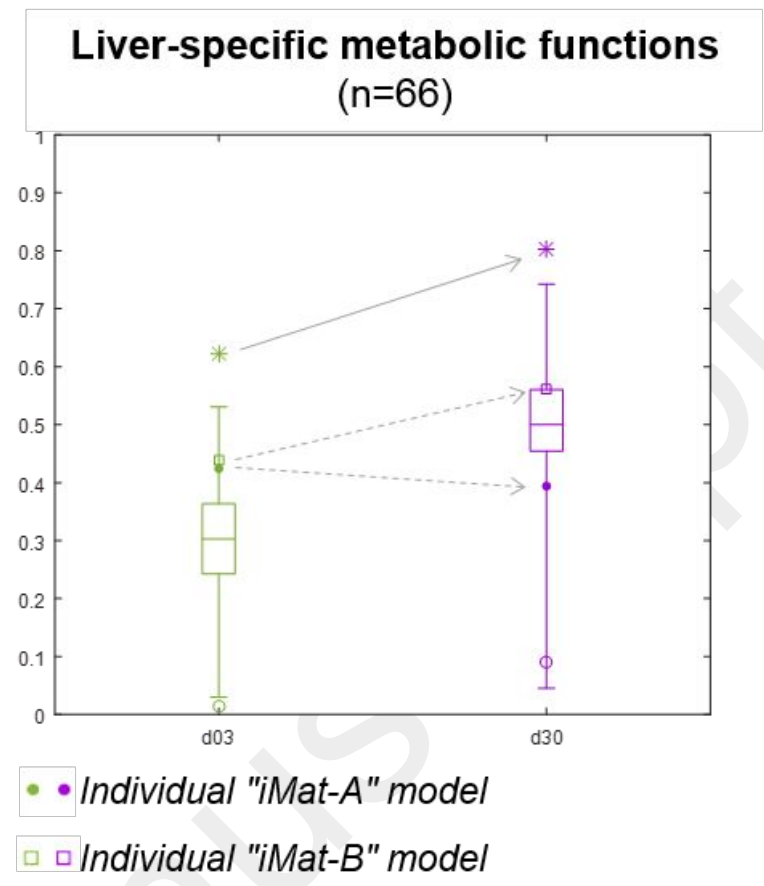
499
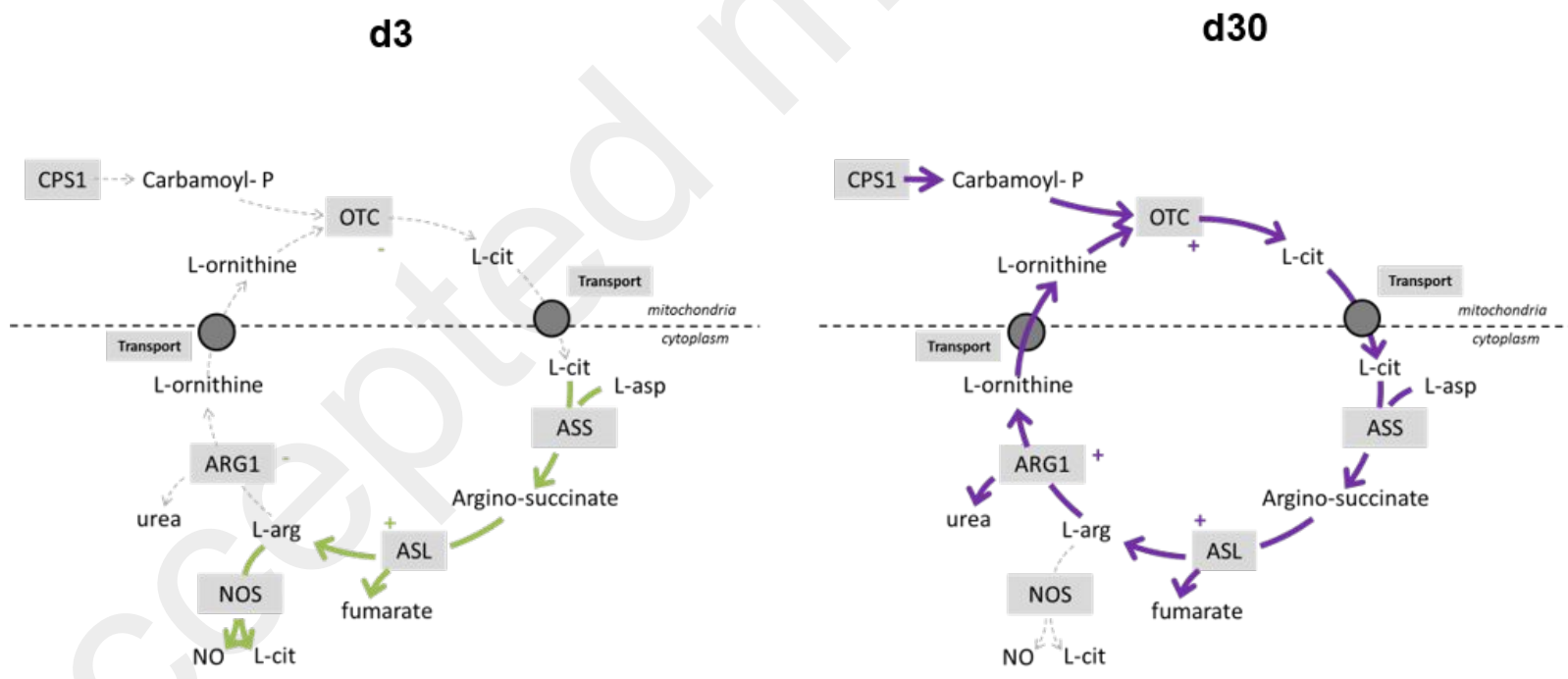

500

+ highly expressed gene

$\rightarrow$ predicted active reaction

- unexpressed gene $-\rightarrow$ predicted inactive reaction

501 Figure 5. Predicted activities for reactions in the urea cycle

502 Table 2. Comparison of liver-specific metabolic functionalities between 3-day and 30-day models 


\section{3-day 30-day}

\begin{tabular}{|c|c|c|}
\hline Bile acid formation & $\mathbf{1}^{\mathrm{a}} / \mathbf{6}^{\mathrm{b}}[0-2]^{\mathrm{c}}$ & $2 / 6[0-6]$ \\
\hline Ureogenesis & $\mathbf{0} / 2[0-0]$ & $\mathbf{2} / 2[0-2]$ \\
\hline gluconeogenesis & $4 / 9 \quad[0-9]$ & $6 / 9 \quad[0-9]$ \\
\hline ketogenesis & $\mathbf{0} / 4 \quad[0-2]$ & $\mathbf{2} / 4 \quad[0-4]$ \\
\hline Amino acid degradation & $\mathbf{1 2} / 23[2-17]$ & $17 / 23 \quad[2-23]$ \\
\hline
\end{tabular}

503

504 We further addressed the ability of progenitor and fully differentiated HepaRG models to account

505 for liver-specificity acquisition, by assessing the proportion (1) of liver-specific genes that were 506 included in each cell-specific subnetwork (based on UNIPROT database annotations, see Material $507 \&$ Methods) and (2) of reactions associated with high confidence level for protein expression in 508 liver (based on the Human Proteome Atlas database information on tissue protein expression, see 509 Material \& Methods). We identified that $46 \%$ of the Recon 2 genes had evidence for expression in 510 liver. On average, a higher proportion of these liver-expressed genes was recalled in the generated 511 subnetworks representing the 30-day cells ( 72 to $84 \%$ depending on the subnetworks) compared to 512 3-day cells (64 to 76\%), consistent with our expectations (Figure S7). We also obtained a higher 513 recall (and proportion) of reactions expressed in hepatocytes with high confidence level, according 514 to the HPA database, in 30-day HepaRG networks (49 to 53\%) compared to 3-day HepaRG 515 networks (36 to 41\%) (Figure S8). Globally, these results tend to validate the ability of our 516 developed models to account for the hepatic specialization occurring during cell-differentiation, 517 when considering conjointly all the cell-specific subnetworks identified for each stage. 518 519 DISCUSSION 
520 The metabolic status of many in vitro models still requires to be duly characterized and this is

521 especially true for models used to study hepatic metabolic diseases, most of which imply, to a 522 certain degree, hepatic regeneration mechanisms. In this study, we propose a methodology, based 523 on large-scale modelling approaches, which enables to point out functional shifts in the global 524 metabolism of cells and apply it to compare the metabolic capacity of hepatic cells at two distinct 525 differentiation stages (progenitor $v$ s. fully differentiated cells). Our developed methodology allows 526 gaining a better understanding of metabolic shifts as it can unveil critical information not directly 527 available from experimental data such as gene expression or metabolomics. Transcriptomic data 528 often provide a restricted coverage of the generic metabolic network genes. In our study, gene 529 expression data covered only $31 \%$ and $32 \%$ of Recon 2 reactions at $\mathrm{d} 3$ and $\mathrm{d} 30$ respectively when 530 considering the genes present in the initial transcriptomic dataset (Figure S3). The problem related 531 to the partial representation of metabolic network genes with commonly used microarrays is an 532 acknowledged fact, with a coverage that varies depending on the type of microarray used ${ }^{48}$. It 533 should also be noted that the partial coverage of metabolic reactions is partly due to a lack of gene 534 related information for some reactions in the network (35\% in Recon 2): these reactions would not 535 be covered whatever the transcriptomic dataset used.. Having such scattered transcriptomic 536 information does not allow to get an overall picture of the metabolic changes occurring during 537 differentiation. By integrating transcriptomic data within the context of a genome-scale metabolic 538 network and computing flux distributions, one takes into account the fact that metabolites used in 539 a reaction need to be produced and consumed, in stoichiometric proportions, by other reactions in 540 the network. Therefore, the activity of a reaction is not only predicted from the expression level of 541 its associated gene but can also be inferred from the activity of downstream and upstream reactions 542 in the network. In our study, $79.2 \%$ and $76.5 \%$ of the reactions predicted to be active at $\mathrm{d} 3$ and $\mathrm{d} 30$ 543 ( $42 \%$ of the reactions if solely considering "required" reactions), respectively, were not associated 
544 with any gene expression data and therefore their activity could be newly predicted thanks to the

545 computational method (Figure S9). A large proportion of these reactions belongs to the transport

546 and exchange pathways, which are less well annotated in term of associated genes, but are,

547 nevertheless, key players in cellular and tissue biology and absolutely need to be taken into account

548 to obtain fully flux-consistent networks. Interestingly, the biotin and tryptophan metabolism

549 pathways, for which gene expression data was available for only a small proportion of the reactions

$550(33-53 \%)$, could not be predicted as activated on the sole basis of transcriptomic data but their

551 predicted activation was inferred by taking into account the network structure and the connections

552 between reactions (Table S5). On the contrary, a few pathways such as "xenobiotics metabolism",

553 "androgen and estrogen synthesis and metabolism" and "arachidonic acid metabolism" were not

554 predicted as activated, although they would be evidenced as activated if basing solely on gene

555 expression data. This is because most of the reactions in these pathways are blocked reactions

556 (Table S5), meaning that their products cannot be further consumed or their substrates cannot be

557 produced. The Recon 2 network contains almost 30\% of "blocked" reactions. Such reactions are

558 always predicted to be inactive when using constraint-based modeling approaches, and can

559 generate false negatives and "artificial" divergences between gene expression data and model

560 predictions that do not have any actual biological meaning. For instance, we observed that $7 \%$ (at

$561 \mathrm{~d} 3$ ) and $8 \%($ at $\mathrm{d} 30)$ of the reactions are predicted as inactive although they are associated with HE

562 genes (Figure S9), but 95 to $99 \%$ of these reactions are actually blocked reactions, meaning that

563 the observed divergence cannot, in this case, be interpreted as a post-regulation process. More

564 interestingly, when not taking into account blocked reactions, about $1 \%$ of the reactions have a

565 predicted activity state that is not in accordance with their associated gene expression data. The

566 discrepancy between data and predictions might also be linked to the presence in the network of

567 promiscuous genes, associated with many different reactions (e.g., the gene EHHADH or hsa:1962 
568 controls 194 reactions). Enforcing a strict translation of the expression or non-expression of such

569 genes to the reaction level would amount to consider that all proteins encoded by a single gene are

570 active (or inactive, respectively) when this gene is expressed (or not/lowly expressed, respectively),

571 which is not biologically relevant. Using the iMat algorithm prevents from doing this strict

572 association, but might consequently generate some discrepancies between predictions and data. In

573 our study, most reactions whose predicted activity is in opposition with their gene expression level

574 belong to extracellular transport and FA oxidation pathways, in which a few genes (respectively

57547 and 93) are associated with a large number of reactions (361 and 709 respectively).

576 Conventional transcriptomic analyses result in lists of identified expressed genes, which are 577 commonly interpreted using pathway enrichment analyses, providing information about which

578 parts of the network are more likely to be differentially expressed in specific experimental 579 conditions ${ }^{49,50}$. However, the definition of metabolic pathways is very dependent on the database

580 or network used and is not always relevant regarding functional metabolism. Indeed, some

581 metabolic functions span over several metabolic pathways, while some metabolic pathways are 582 very generic and encompass several distinct metabolic functions ${ }^{51}$. Thus, one may miss a specific 583 or biologically relevant functionality when relying on these global pathways for interpretation.

584 Generating flux-consistent models of the functional metabolic network of cells offers the clear 585 advantage of going beyond the analysis of metabolic pathways by enabling a more holistically 586 identification of modulated metabolic functions and therefore providing a finer assessment of the 587 metabolic capacities of a system. For instance, in our study, we predicted that ureagenesis is 588 activated during the differentiation process, although the "urea cycle" pathway was not evidenced 589 as activated if relying only on the pathway enrichment analysis ( $p$-value $=1$ ). Indeed, in Recon 2, 590 the "urea cycle pathway" encompasses 69 reactions, but only a few of them are truly involved in 591 urea synthesis (other reactions contribute to creatine or spermidine metabolism) so that the 
592 predicted activation of these reactions was masked by the lack of changes of many other reactions

593 in this pathway.

594 The originality of the approach we propose in this study mainly relies on the fact that we chose

595 not to arbitrarily select one specific model among all equally optimal cell-specific models

596 identified, but rather to consider them conjointly for each differentiation stage. We observed that

597 the existence of a high number of alternative possible subnetworks is partly due to the existence of

598 redundant reactions in the initial generic reconstruction that often reflect artifactitious redundancies

599 rather than a true biological alternative. Manual curation of these redundancies would contribute

600 to decrease the number of computed equally adequate stage-specific models and therefore reduce

601 the uncertainty in the prediction of reaction activity. Despite this well identified issue, we showed

602 that the comparison of sets of several similarly adequate subnetworks for two distinct conditions

603 still enables evidencing stage-dependent differences, as stage-dependent differences contributed

604 more to the discrimination among all subnetworks than the variability between the subnetworks at

605 each stage (Figure 2). Other methods used to generate cell-specific metabolic models have adopted

606 a different strategy, which consists in selecting one specific solution which optimizes an additional

607 criteria, such as minimizing the total sum of fluxes in the network (Euclidean FBA), maximizing

608 the biomass production or cell growth ${ }^{52}$, minimizing the number of active reactions ${ }^{39}$ or being

609 able to achieve a set of predefined metabolic tasks ${ }^{14}$. This entails to make some additional and $a$

610 priori assumptions about the "optimal" metabolic state of the cells or some target metabolic

611 functions. This can be relevant in the case of rapidly growing cells, such as cancer cells. However,

612 it is much less relevant for cells that are not in a permanent proliferative status and are able to

613 achieve a wide span of metabolic activities, such as liver cells. We assessed the benefit of our

614 approach by comparing our results with results obtained when considering only one subnetwork

615 for each stage, either arbitrarily selected among all the subnetworks identified by the iMat 
616 algorithm (iMat-A and iMat-B, in Figure 4 \& Figures S7 \& S8) or generated using the FASTCORE

617 algorithm ${ }^{39}$ (see Material and Methods). When considering single solutions, we observed that

618 some metabolic pathways, with little gene expression information, appeared as significantly

619 enriched in activated (e.g., ubiquinone synthesis) or inactivated reactions (e.g., keratan sulfate

620 degradation, $\mathrm{N}$-glycan synthesis) solely as a result of arbitrariness in the choice of the subnetwork

621 at each stage (Table S6), whereas the method developed in this work prevents from making such

622 biased predictions. For instance, the ubiquinone synthesis pathway (Figure S10) was predicted as

623 activated when using the FASTCORE algorithm or considering only one iMat solution. Because

624 no gene-expression information is available at $\mathrm{d} 3$ for any of the reactions in this pathway, all these

625 reactions can be predicted to be either active or inactive at $\mathrm{d} 3$. As a result and because reactions

626 are necessarily active at $\mathrm{d} 30$, whatever the selected subnetwork, it may then be concluded that the

627 pathway is activated if reactions are predicted to be inactive in the retained $\mathrm{d} 3$-subnetwork, which

628 is what happens when using the FASTCORE algorithm that aims to minimize the number of

629 reactions (Figure S10C\&D). Conversely, with the strategy we implemented in our method, which

630 takes into consideration all the possibilities of reactions activity at $\mathrm{d} 3$, we do not predict that this

631 pathway is activated (Figure S10B), retaining a more cautious conclusion. The same interpretations

632 can be made for the N-glycan synthesis and keratin sulfate degradation pathways (Figures S11 \&

633 S12 and Supplementary Notes). The FASTCORE algorithm provides one unique minimal

634 subnetwork, which contains all the reactions associated with highly expressed genes and a minimal

635 set of additional reactions required in order to get a flux-consistent network. Although it might

636 reduce the bias in the comparison of the conditions by providing a deterministic way to define one

637 unique model for each condition, we suspect that these minimal networks are more dependent on

638 the transcriptomic data and are likely biased toward assuming that reactions are not active when

639 they have no associated expression data. In addition, it is questionable whether the metabolic state 
640 involving a minimal number of biochemical reactions as computed by the FASTCORE algorithm

641 is truly a good picture of the actual metabolic capacity of a mammalian cell. We reached the

642 conclusion that, in the case of insufficient experimental data, arbitrarily selecting one possible

643 subnetwork could lead to erroneous conclusions and we argue that our method, which takes into

644 account and compares sets of equally optimal subnetworks, prevents from making potentially false

645 positive, and therefore erroneous, predictions regarding the activation or inactivation of pathways.

646 Getting different results when using different algorithms for identifying cell-specific models is a

647 well acknowledged issue, which has already been reported in several studies ${ }^{53,54}$. No method

648 provides more accurate or exact results than others, and the choice of the method should be made

649 depending on the available experimental data, as well as the context and the aim of the study.

650 However, the approach that we propose here has the advantage not to require any other information

651 than transcriptomic data and does not make any biological assumption about the metabolic state or

652 objective of the cell system. For this reason, it can interestingly be applied to poorly characterized

653 cell systems. Such approaches are nowadays absolutely relevant and necessary for a sound and

654 untargeted assessment of metabolic modulations because they allow for an accurate modeling of

655 cellular metabolic networks out of omics data with a minimal number of a priori assumptions and

656 therefore limit the risk of false-predictions. Also, considering all adequately possible subnetworks

657 seem relevant under the hypothesis that the variability observed between the distinct possible

658 subnetworks partly reflects the actual metabolic heterogeneity in a population of cells. Therefore,

659 taking into consideration this variability should provide a better picture of the whole metabolic 660 capability of heterogeneous cellular systems.

661

662 CONCLUSION 
663 In conclusion, by implementing a new strategy integrating transcriptomic and metabolomic data 664 within the context of the global human genome-scale metabolic model, we were able to better 665 characterize the metabolic state of HepaRG cells at two stages of differentiation and to identify 666 some metabolic functions that are set up during the differentiation process of this human hepatic 667 model. Our predictions are consistent with the known activation of pathways corresponding to 668 hepato-specific functionalities (bile acid synthesis, cytochrome metabolism) during the 669 differentiation process, but also newly evidenced the modulation of other metabolic pathways, 670 involving the metabolism of biotin, tryptophan and FAs, which could not have been evidenced 671 solely from transcriptomic data. The approach we applied in this study offers several advantages 672 compared to both analyses based on transcriptomic data and common computational approaches 673 used to identify cell- or tissue-specific metabolic models: it especially allows to point out metabolic 674 pathways which do not have gene annotations, to go further the analysis of traditionally defined 675 metabolic pathways by identifying modulated metabolic functions, and to reduce the risk of false 676 predictions by retaining a set of networks instead of a particular (sometime arbitrarily defined) one.

677 The result is that it allows a comprehensive and functional assessment of the metabolic capacity of 678 cells with a minimal number of a priori assumptions. It opens interesting potential for the 679 comparison of global metabolic shifts occurring during differentiation process of various cell types, 680 but also in the broader context of long-term metabolic changes, such as induced during the onset 681 of chronic metabolic diseases or chronic exposure to toxics. 
682

683

684

685

686

687

688

689

690

691

692

693

694

695

696

697

698

699

700

701

702

703

\section{FIGURES}

Figure 1. Predicted activity of Recon 2 reactions in stage-specific subnetworks. (A) The activity of Recon 2 reactions was predicted using the iMat algorithm. Several solutions were found and the reactions were classified as "required" (R) if they were found to be active in all solutions and conversely "Inactive" (I) if they display a zero-flux in all the optimal solutions. All other reactions, found to be either active or inactive in the optimal solutions, were classified as "Potentially active" (PA). This analysis was made independently for the $\mathrm{d} 3$ and $\mathrm{d} 30$ differentiation stages. (B) Reactions identified as I at $\mathrm{d} 3$ but $\mathrm{R}$ or PA at $\mathrm{d} 30$ were considered as activated, whereas reactions identified as $\mathrm{R}$ or $\mathrm{PA}$ at $\mathrm{d} 3$ but $\mathrm{I}$ at $\mathrm{d} 30$ were considered as activated.

Figure 2. Principal component analysis of 3-day and 30-day subnetworks. PCA analysis was performed on the whole set of equally adequate subnetworks obtained for both stages $(n=6847)$. Reactions were used as variables. Each subnetwork was represented as a binary vector with 0 values for inactive reactions and 1 values for active reactions.

Figure 3. Visualization of predicted modulated reactions in the fatty acid oxidation pathway. Reactions belonging to the fatty acid oxidation pathway in Recon 2 were extracted and visualized using the MetExplore webserver (Chazalviel et al., 2018). Reactions predicted to be activated or inactivated between the 2 differentiation stages were mapped onto the pathway.

Figure 4. Simulations of metabolic functions in 3-day and 30-day models. Metabolic functions were simulated for each of the cell-specific models identified at each stage and for the model obtained using the FASTCORE algorithm. Boxplots represent the distribution of the proportion of simulated functions that can be achieved by 3-day and 30-day cell specific models for all generic (left panel) or all liver-specific (right panel) metabolic functions. UNION of cell-specific models, 
704 represented by a star, corresponds to the proportion of functions that can be achieved by at least 705 one of the cell-specific model. The iMat-A and iMat-B points correspond to the proportion of 706 functions that can be achieved by these 2 randomly selected subnetworks individually. Arrows 707 represent the sense of variation between $\mathrm{d} 3$ and $\mathrm{d} 30$, which can be different depending on which 708 subnetworks we compare.

709 Figure 5. Predicted activities for reactions in the urea cycle. Reactions predicted to be active 710 are represented with green plain arrows for $\mathrm{d} 3$ and purple plain arrows for $\mathrm{d} 30 . "+"$ and "-" signs

711 indicate reactions associated with highly or unexpressed genes. The following reactions belonging 712 to the urea cycle are represented (names in italic correspond to the reaction identifiers in Recon2):

713 CPS1, carbamoyl-phosphate synthase (CBPSam \& r0034); OTC, ornithine carbamoyltransferase 714 (OCBTm); ASS, argininosuccinate synthase (ARGSS); ASL, argininosuccinate lyase (ARGSL);

715 ARG1, arginase $(A R G N)$; NOS, L-Arginine,NADPH:oxygen oxidoreductase (NO-forming) 716 (r0145); ornithine mitochondrial transport exchange with citruline (ORNt4m) (grey circle).

720 Table 1. Predictions of metabolic pathways significantly modulated between $\mathrm{d} 3$ and $\mathrm{d} 30$

\begin{tabular}{ll}
\hline ACTIVATED pathways & $\begin{array}{l}\text { corrected } \\
\text { p-value }\end{array}$ \\
Pathways & $1.31 \mathrm{e}^{-11}$ \\
\hline Biotin metabolism & $6.80 \mathrm{e}^{-07}$ \\
Fatty acid oxidation & $5.13 \mathrm{e}^{-05}$ \\
Bile acid synthesis & $2.44 \mathrm{e}^{-04}$ \\
Tryptophan metabolism & $3.76 \mathrm{e}^{-04}$ \\
Cytochrome metabolism &
\end{tabular}

INACTIVATED pathways

\begin{tabular}{ll} 
Pathways & $\begin{array}{l}\text { corrected } \\
\mathrm{p} \text {-value }\end{array}$ \\
\hline Transport, extracellular & $5.60 \mathrm{e}^{-31}$ \\
Fatty Acid Synthesis & $1.30 \mathrm{e}^{-15}$ \\
&
\end{tabular}



Blood group synthesis
$1.59 \mathrm{e}^{-03}$
Lysine metabolism
$1.44 \mathrm{e}^{-03}$
Limonene \& pinene degradation $3.45 \mathrm{e}^{-02}$

721

722

723

724

725
726

727

728

729

730

731

732

733

734

735

736

737

738

Pathway enrichment analysis was performed on set of reactions identified as inactivated $(\mathrm{n}=200)$ or activated $(\mathrm{n}=484)$ between 3 -day and 30-day cell models, after removing blocked reactions. Pvalues were obtained by performing a hypergeometric test followed by a Bonferroni correction.

Table 2. Comparison of liver-specific metabolic functionalities between 3-day and 30-day models

\begin{tabular}{|c|c|c|}
\hline & d3 & d30 \\
\hline Bile acid formation & $\mathbf{1}^{\mathrm{a} / 6^{\mathrm{b}}}[0-2]^{\mathrm{c}}$ & $2 / 6[0-6]$ \\
\hline Ureogenesis & $\mathbf{0} / 2[0-0]$ & $2 / 2 \quad[0-2]$ \\
\hline gluconeogenesis & $4 / 9 \quad[0-9]$ & $6 / 9 \quad[0-9]$ \\
\hline ketogenesis & $\mathbf{0} / 4 \quad[0-2]$ & $2 / 4 \quad[0-4]$ \\
\hline Amino acid degradation & $12 / 23[2-17]$ & $17 / 23 \quad[2-23]$ \\
\hline
\end{tabular}

Results from liver-specific metabolic functions simulated for each of the cell-specific models identified at each stage: for each type of function, values in bold (a) indicate the average number of simulated functions that can be achieved out of the total number of tested functions (b). Values in square brackets (c) correspond to the minimal and maximal numbers of simulated functions that can be achieved.

\section{ASSOCIATED CONTENT}

\section{Supporting information.}

Principle of the iMat algorithm illustrated on a Toy model (Figure S1); Workflow of analyses implemented for comparison of stage-specific subnetworks (Figure S2); Distribution of genes expression levels in experimental data set and Recon 2 network (Figure S3); Comparison of predicted active reactions in subnetworks between the 2 stages (Figure S4); Analysis of heterogeneity in 30-day subnetworks (Figure S5); Visualization of predicted modulated reactions 
739 in the fatty acid synthesis pathway (Figure S6); Assessment of the liver-specificity of generated

740 subnetworks: comparison with UNIPROT data (Figure S7); Assessment of the liver-specificity of

741 generated subnetworks: comparison with Human Proteome Atlas data (Figure S8); Comparison of

742 predicted reaction activity with gene expression data (Figure S9); Comparison between predictions

743 made from conjoint or individual analysis of cell-specific models for the ubiquinone synthesis

744 pathway (Figure S10); Comparison between predictions made from conjoint or individual analysis

745 of cell-specific models for the N-glycan synthesis pathway (Figure S11); Comparison between

746 predictions made from conjoint or individual analysis of cell-specific models for the keratin sulfate

747 degradation pathway (Figure S12); Adequacy of model predictions with gene expression data

748 (Table S3); Modulated pathways identified using pathway enrichment: comparison between

749 predictions made from generated models \& transcriptomic data (Table S5); Modulated pathways

750 identified using pathway enrichment: comparison between predictions made from conjoint or

751 individual analysis of cell-specific models (Table S6). Interpretations of activated or inactivated

752 reactions depending on the selected optimization solution for the Ubiquinone, $\mathrm{N}$-glycan synthesis

753 and keratin sulfate degradation pathways (Supplementary Notes) (PDF)

754 Metabolic functions used for simulations (Table S1, XLSX)

755 NMR identified metabolites for 3-day and 30-day HepaRG cells (Table S2, XLSX)

756 Computed subnetworks for 3-day and 30-day HepaRG cells from our method and the

757 FASTCORE algorithm (Table S4, XLSX)

758

759 This material is available free of charge via the Internet at http://pubs.acs.org. 
761 AUTHOR INFORMATION

762 Present adress

$763 \uparrow$ SRSMC, UMR 7585, Université de Lorraine, France. \$INSB Institut des sciences biologiques, 764 UMR8204 Centre d'infection et d'immunité de Lille, France.

\section{Author contributions}

766 FJ, NP, AC, FMo and MAR designed the research; FMo, HDS, CC, SB, EP and NC performed the

767 experiments; NP developed the method and performed the computational analysis; FMa, CF and

768 FV participated in the computational analysis; NP wrote the manuscript; FJ, DZ, BF, NC, AC and

769 NP contributed in data interpretation, discussion on results and writing and revising the manuscript.

\section{$770 \quad$ Funding source}

771 This work was supported by the French Ministry of Research and National Research Agency as 772 part of the French MetaboHUB, the national metabolomics and fluxomics infrastructure (Grant 773 ANR-INBS-0010) and the NISTEC project (Grant ANR-09-CESA-003).

775 ACKNOWLEDGMENT

776 This work was supported in part by the French Ministry of Research and the National Research 777 Agency, as part of the French MetaboHUB infrastructure (the national metabolomics and 778 fluxomics infrastructure, Grant ANR-INBS-0010) and the NISTEC project (Grant ANR-09779 CESA-003). The authors thank Maxime Chazalviel for its work on metabolic network visualization 780 and Dr Marc Audebert for providing valuable help on experimental procedures. 


\section{REFERENCES}

783 (1) Thiele, I.; Palsson, B. Ø. Reconstruction Annotation Jamborees: A Community Approach to Systems Biology. Mol.Svst. Biol. 2010, 6, 361.

785 (2) Duarte, N. C.; Becker, S. A.; Jamshidi, N.; Thiele, I.; Mo, M. L.; Vo, T. D.; Srivas, R.;

786 Palsson, B. Ø. Global Reconstruction of the Human Metabolic Network Based on Genomic and Bibliomic Data. Proc. Natl. Acad. Sci. U. S. A. 2007, 104 (6), 1777-1782.

788 (3) Ma, H.; Sorokin, A.; Mazein, A.; Selkov, A.; Selkov, E.; Demin, O.; Goryanin, I. The 789 Edinburgh Human Metabolic Network Reconstruction and Its Functional Analysis. Mol Syst Biol 2007, 3, 135.

791 (4) Mardinoglu, A.; Agren, R.; Kampf, C.; Asplund, A.; Uhlen, M.; Nielsen, J. Genome-Scale 792 Metabolic Modelling of Hepatocytes Reveals Serine Deficiency in Patients with NonAlcoholic Fatty Liver Disease. Nat Commun 2014, 5 (May 2013), 3083.

794 (5) Thiele, I.; Swainston, N.; Fleming, R. M. T.; Hoppe, A.; Sahoo, S.; Aurich, M. K.; 795 Haraldsdottir, H.; Mo, M. L.; Rolfsson, O.; Stobbe, M. D.; et al. A Community-Driven 796 Global Reconstruction of Human Metabolism. Nat Biotechnol 2013, 31 (5), 419-425.

797 (6) Smallbone, K. Striking a Balance with Recon 2.1. 2013.

798 (7) Swainston, N.; Smallbone, K.; Hefzi, H.; Dobson, P. D.; Brewer, J.; Hanscho, M.; Zielinski, 799 D. C.; Ang, K. S.; Gardiner, N. J.; Gutierrez, J. M.; et al. Recon 2.2: From Reconstruction $800 \quad$ to Model of Human Metabolism. Metabolomics 2016, 12 (7), 109.

801 (8) Blais, E. M.; Rawls, K. D.; Dougherty, B. V; Li, Z. I.; Kolling, G. L.; Ye, P.; Wallqvist, A.; 
802 Papin, J. A. Reconciled Rat and Human Metabolic Networks for Comparative 803 Toxicogenomics and Biomarker Predictions. Nat. Commun. 2017, 8, 14250.

804 (9) Brunk, E.; Sahoo, S.; Zielinski, D. C.; Altunkaya, A.; Dräger, A.; Mih, N.; Gatto, F.; Nilsson, 805 A.; Preciat Gonzalez, G. A.; Aurich, M. K.; et al. Recon3D Enables a Three-Dimensional 806 View of Gene Variation in Human Metabolism. Nat. Biotechnol. 2018, 36 (3), $272-281$.

807 (10) Shlomi, T.; Cabili, M. N.; Herrgard, M. J.; Palsson, B. Ø. O.; Ruppin, E.; Herrgård, M. J.; 808 Palsson, B. Ø. O.; Ruppin, E. Network-Based Prediction of Human Tissue-Specific 809 Metabolism. Nat Biotech 2008, 26 (9), 1003-1010.

810 (11) Jerby, L.; Shlomi, T.; Ruppin, E. Computational Reconstruction of Tissue-Specific 811 Metabolic Models: Application to Human Liver Metabolism. Mol Syst Biol 2010, 6, 401.

812 (12) Bordbar, A.; Jamshidi, N.; Palsson, B. O. IAB-RBC-283: A Proteomically Derived 813 Knowledge-Base of Erythrocyte Metabolism That Can Be Used to Simulate Its 814 Physiological and Patho-Physiological States. BMC Syst Biol 2011, 5, 110.

815 (13) Agren, R.; Bordel, S.; Mardinoglu, A.; Pornputtapong, N.; Nookaew, I.; Nielsen, J. 816 Reconstruction of Genome-Scale Active Metabolic Networks for 69 Human Cell Types and 817 Cancer Types Using INIT. PLoS Comput Biol 2012, 8 (5), e1002518.

818 (14) Agren, R.; Mardinoglu, A.; Asplund, A.; Kampf, C.; Uhlen, M.; Nielsen, J. Identification of 819 Anticancer Drugs for Hepatocellular Carcinoma through Personalized Genome-Scale $820 \quad$ Metabolic Modeling. Mol. Syst. Biol. 2014, 10, 1-13.

821 (15) Frezza, C.; Zheng, L.; Folger, O.; Rajagopalan, K. N.; MacKenzie, E. D.; Jerby, L.; 822 Micaroni, M.; Chaneton, B.; Adam, J.; Hedley, A.; et al. Haem Oxygenase Is Synthetically 
823 Lethal with the Tumour Suppressor Fumarate Hydratase. Nature 2011, 477 (7363), 225824228.

825 (16) Goldstein, I.; Yizhak, K.; Madar, S.; Goldfinger, N.; Ruppin, E.; Rotter, V. P53 Promotes 826 the Expression of Gluconeogenesis-Related Genes and Enhances Hepatic Glucose 827 Production. Cancer Metab. 2013, 1 (1), 9.

828 (17) Yizhak, K.; Le Dévédec, S. E.; Rogkoti, V. M.; Baenke, F.; de Boer, V. C.; Frezza, C.; 829 Schulze, A.; van de Water, B.; Ruppin, E. A Computational Study of the Warburg Effect 830 Identifies Metabolic Targets Inhibiting Cancer Migration. Mol. Syst. Biol. 2014, 10, 744.

831 (18) Orth, J. D.; Thiele, I.; Palsson, B. Ø. O. What Is Flux Balance Analysis? Nat Biotechnol $832 \quad \mathbf{2 0 1 0}, 28(3), 245-248$.

833 (19) Zur, H.; Ruppin, E.; Shlomi, T. IMAT: An Integrative Metabolic Analysis Tool. 834 Bioinformatics 2010, $26(24), 3140-3142$.

835 (20) Aninat, C.; Piton, A.; Glaise, D.; Le Charpentier, T.; Langouët, S.; Morel, F.; Guguen836 Guillouzo, C.; Guillouzo, A. Expression of Cytochrome P450 Enzymes and Nuclear 837 Receptors in Human Hepatoma HepaRG Cells. Drug Metab. Dispos. 2006, 34 (1), 75-83.

838 (21) Rodrigues, R. M.; Heymans, A.; De Boe, V.; Sachinidis, A.; Chaudhari, U.; Govaere, O.; 839 Roskams, T.; Vanhaecke, T.; Rogiers, V.; De Kock, J. Toxicogenomics-Based Prediction of 840 Acetaminophen-Induced Liver Injury Using Human Hepatic Cell Systems. Toxicol. Lett. $841 \quad 2016,240(1), 50-59$.

842 (22) Peyta, L.; Jarnouen, K.; Pinault, M.; Guimaraes, C.; Pais de Barros, J.-P.; Chevalier, S.; 843 Dumas, J.-F.; Maillot, F.; Hatch, G. M.; Loyer, P.; et al. Reduced Cardiolipin Content 
844 Decreases Respiratory Chain Capacities and Increases ATP Synthesis Yield in the Human 845 HepaRG Cells. Biochim. Biophys. Acta - Bioenerg. 2016, 1857 (4), 443-453.

846 (23) Ma, X.; Duan, Y.; Tschudy-Seney, B.; Roll, G.; Behbahan, I. S.; Ahuja, T. P.; Tolstikov, V.; 847 Wang, C.; McGee, J.; Khoobyari, S.; et al. Highly Efficient Differentiation of Functional 848 Hepatocytes From Human Induced Pluripotent Stem Cells. Stem Cells Transl. Med. 2013, 2 $849 \quad(6), 409-419$.

850 (24) Zhang, J.; Nuebel, E.; Daley, G. Q.; Koehler, C. M.; Teitell, M. A. Metabolic Regulation in 851 Pluripotent Stem Cells during Reprogramming and Self-Renewal. Cell Stem Cell 2012, 11 $852 \quad(5), 589-595$.

853 (25) Xu, X.; Duan, S.; Yi, F.; Ocampo, A.; Liu, G.-H.; Izpisua Belmonte, J. C. Mitochondrial 854 Regulation in Pluripotent Stem Cells. Cell Metab. 2013, 18 (3), 325-332.

855 (26) Teslaa, T.; Teitell, M. A. Pluripotent Stem Cell Energy Metabolism: An Update. EMBO J. $856 \quad 2015,34(2), 138-153$.

857 (27) Bucher, S.; Jalili, P.; Le Guillou, D.; Begriche, K.; Rondel, K.; Martinais, S.; Zalko, D.; 858 Corlu, A.; Robin, M.-A.; Fromenty, B. Bisphenol a Induces Steatosis in HepaRG Cells 859 Using a Model of Perinatal Exposure. Environ. Toxicol. 2017, 32 (3), 1024-1036.

860 (28) Cerec, V.; Glaise, D.; Garnier, D.; Morosan, S.; Turlin, B.; Drenou, B.; Gripon, P.; 861 Kremsdorf, D.; Guguen-Guillouzo, C.; Corlu, A. Transdifferentiation of Hepatocyte-like 862 Cells from the Human Hepatoma HepaRG Cell Line through Bipotent Progenitor. 863 Hepatology 2007, 45, 957-967.

864 (29) Hart, S. N.; Li, Y.; Nakamoto, K.; Subileau, E. a.; Steen, D.; Zhong, X. b. A Comparison of 
865

866

867

868

869

870

871

872

873

874

875

876

877

878

879

880

881

882

883

884

885

Whole Genome Gene Expression Profiles of HepaRG Cells and HepG2 Cells to Primary Human Hepatocytes and Human Liver Tissues. Drug Metab. Dispos. 2010, 38 (6), 988-994.

(30) Parent, R.; Beretta, L. Translational Control Plays a Prominent Role in the Hepatocytic Differentiation of HepaRG Liver Progenitor Cells. Genome Biol. 2008, 9, R19.

(31) Wishart, D. S.; Jewison, T.; Guo, A. C.; Wilson, M.; Knox, C.; Liu, Y.; Djoumbou, Y.; Mandal, R.; Aziat, F.; Dong, E.; et al. HMDB 3.0-The Human Metabolome Database in 2013. Nucleic Acids Res. 2012, 41 (D1), D801-D807.

(32) Rossell, S.; Huynen, M. A.; Notebaart, R. A. Inferring Metabolic States in Uncharacterized Environments Using Gene-Expression Measurements. PLoS Comput Biol 2013, 9 (3), e1002988.

(33) UniProt: The Universal Protein Knowledgebase. Nucleic Acids Res. 2017, 45 (D1), D158D169.

(34) Uhlen, M.; Fagerberg, L.; Hallstrom, B. M.; Lindskog, C.; Oksvold, P.; Mardinoglu, A.; Sivertsson, A.; Kampf, C.; Sjostedt, E.; Asplund, A.; et al. Tissue-Based Map of the Human Proteome. Science (80-. ). 2015, 347 (6220), 1260419-1260419.

(35) Dennis, G.; Sherman, B. T.; Hosack, D. A.; Yang, J.; Gao, W.; Lane, H.; Lempicki, R. A.; Quackenbush, J.; Wheeler, D.; Chappey, C.; et al. DAVID: Database for Annotation, Visualization, and Integrated Discovery. Genome Biol. 2003, 4 (9), R60.

(36) Bordbar, A.; Feist, A. M.; Usaite-Black, R.; Woodcock, J.; Palsson, B. O.; Famili, I. A Multi-Tissue Type Genome-Scale Metabolic Network for Analysis of Whole-Body Systems Physiology. BMC Syst Biol 2011, 5, 180. 
886 (37) Gille, C.; Bolling, C.; Hoppe, A.; Bulik, S.; Hoffmann, S.; Hubner, K.; Karlstadt, A.; 887 Ganeshan, R.; Konig, M.; Rother, K.; et al. HepatoNet1: A Comprehensive Metabolic 888 Reconstruction of the Human Hepatocyte for the Analysis of Liver Physiology. Mol Syst $889 \quad$ Biol 2010, $6(411), 411$.

890 (38) Cottret, L.; Wildridge, D.; Vinson, F.; Barrett, M. P.; Charles, H.; Sagot, M. F.; Jourdan, F. 891 MetExplore: A Web Server to Link Metabolomic Experiments and Genome-Scale 892 Metabolic Networks. Nucleic Acids Res 2010, 38 (Web Server issue), W132-7.

893 (39) Vlassis, N.; Pacheco, M. P.; Sauter, T. Fast Reconstruction of Compact Context-Specific 894 Metabolic Network Models. arXiv:1304.7992 [q-bio.MN] 2013, 10, 1-15.

895 (40) Tong, L. Structure and Function of Biotin-Dependent Carboxylases. Cell. Mol. Life Sci. 896 2013, $70(5), 863-891$.

897 (41) Badawy, A. A.-B. Tryptophan Metabolism, Disposition and Utilization in Pregnancy. $898 \quad$ Biosci. Rep. 2015, 35 (5), e00261-e00261.

899 (42) Suda, T.; Robinson, J. C.; Fjellstedt, T. A. Developmental Changes in the Enzymatic 900 Capacity for Reduction and Oxidation of Alpha-Ketoadipate in Rat Liver, Heart, Kidney, $901 \quad$ and Brain. Pediatr. Res. 1978, 12 (4 Pt 1), 297-300.

902 (43) Chazalviel, M.; Frainay, C.; Poupin, N.; Vinson, F.; Merlet, B.; Gloaguen, Y.; Cottret, L.; 903 Jourdan, F. MetExploreViz: Web Component for Interactive Metabolic Network $904 \quad$ Visualization. Bioinformatics 2018, 34 (2), 312-313.

905 (44) Wanders, R. J. A.; Waterham, H. R.; Ferdinandusse, S. Metabolic Interplay between 906 Peroxisomes and Other Subcellular Organelles Including Mitochondria and the 
907 Endoplasmic Reticulum. Front. Cell Dev. Biol. 2016, 3, 83.

908 (45) Kersten, S. Integrated Physiology and Systems Biology of PPARa. Mol. Metab. 2014, 3 (4), $909 \quad 354-371$.

910 (46) Fan, C. Y.; Pan, J.; Chu, R.; Lee, D.; Kluckman, K. D.; Usuda, N.; Singh, I.; Yeldandi, A. 911 V; Rao, M. S.; Maeda, N.; et al. Hepatocellular and Hepatic Peroxisomal Alterations in Mice 912 with a Disrupted Peroxisomal Fatty Acyl-Coenzyme A Oxidase Gene. J. Biol. Chem. 1996, $913 \quad 271(40), 24698-24710$.

914 (47) Newman, J. C.; Verdin, E. $\beta$-Hydroxybutyrate: A Signaling Metabolite. Annu. Rev. Nutr. $915 \quad 2017,37(1), 51-76$.

916 (48) Zelezniak, A.; Pers, T. H.; Soares, S.; Patti, M. E.; Patil, K. R. Metabolic Network Topology 917 Reveals Transcriptional Regulatory Signatures of Type 2 Diabetes. PLoS Comput. Biol. $918 \quad$ 2010, $6(4)$, e 1000729.

919 (49) Chagoyen, M.; Pazos, F. MBRole: Enrichment Analysis of Metabolomic Data. $920 \quad$ Bioinformatics 2011, 27 (5), 730-731.

921 (50) Persicke, M.; Rückert, C.; Plassmeier, J.; Stutz, L. J.; Kessler, N.; Kalinowski, J.; Goesmann, 922 A.; Neuweger, H. MSEA: Metabolite Set Enrichment Analysis in the MeltDB Metabolomics 923 Software Platform: Metabolic Profiling of Corynebacterium Glutamicum as an Example. $924 \quad$ Metabolomics 2012, 8 (2), 310-322.

925 (51) Frainay, C.; Jourdan, F. Computational Methods to Identify Metabolic Sub-Networks Based 926 on Metabolomic Profiles. Brief. Bioinform. 2017, 18 (1), 43-56.

927 (52) Becker, S. A.; Palsson, B. O. Context-Specific Metabolic Networks Are Consistent with 
928 Experiments. PLoS Comput. Biol. 2008, 4 (5), e1000082.

929 (53) Machado, D.; Herrgård, M. Systematic Evaluation of Methods for Integration of 930 Transcriptomic Data into Constraint-Based Models of Metabolism. PLoS Comput. Biol. $931 \quad 2014,10(4)$, e 1003580.

932 (54) Pacheco, M. P.; Pfau, T.; Sauter, T. Benchmarking Procedures for High-Throughput Context 933 Specific Reconstruction Algorithms. Front. Physiol. 2016, 6, 410.

934

935

936 For Table of Content only

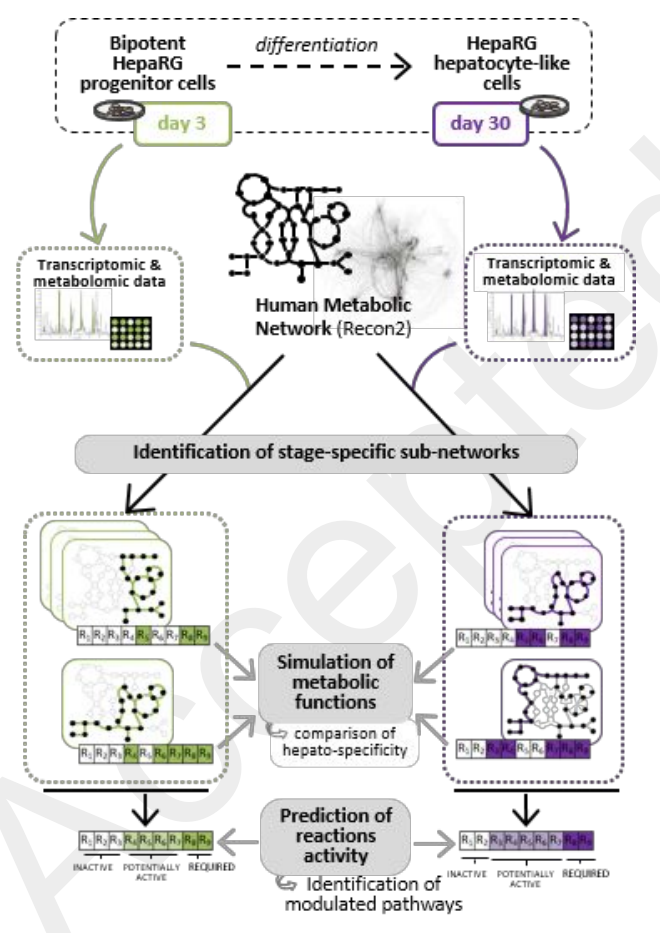

